\title{
Dynamic-stall measurements using time-resolved pressure-sensitive paint on double-swept rotor blades
}

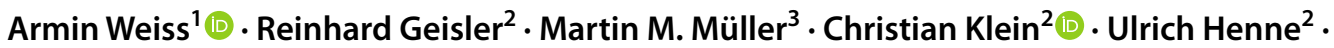 \\ Johannes N. Braukmann² ${ }^{\mathbb{D}}$. Johannes Letzgus ${ }^{4}$
}

Received: 20 August 2021 / Revised: 10 November 2021 / Accepted: 6 December 2021 / Published online: 23 December 2021

(C) The Author(s) 2021

\begin{abstract}
The study presents an optimized pressure-sensitive paint (PSP) measurement system that was applied to investigate unsteady surface pressures on recently developed double-swept rotor blades in the rotor test facility at the German Aerospace Center (DLR) in Göttingen. The measurement system featured an improved version of a double-shutter camera that was designed to reduce image blur in PSP measurements on fast rotating blades. It also comprised DLR's PSP sensor, developed to capture transient flow phenomena (iPSP). Unsteady surface pressures were acquired across the outer $65 \%$ of the rotor blade with iPSP and at several radial blade sections by fast-response pressure transducers at blade-tip Mach and Reynolds numbers of $\mathrm{M}_{\text {tip }}=0.282-0.285$ and $\mathrm{Re}_{\text {tip }}=5.84-5.95 \times 10^{5}$. The unique experimental setup allowed for scanning surface pressures across the entire pitch cycle at a phase resolution of $0.225 \mathrm{deg}$ azimuth for different collective and cyclic-pitch settings. Experimental results of both investigated cyclic-pitch settings are compared in detail to a delayed detached eddy simulation using the flow solver FLOWer and to flow visualizations from unsteady Reynolds-averaged Navier-Stokes (URANS) computations with DLR's TAU code. The findings reveal a detailed and yet unseen insight into the pressure footprint of double-swept rotor blades undergoing dynamic stall and allow for deducing "stall maps", where confined areas of stalled flow on the blade are identifiable as a function of the pitch phase.
\end{abstract}

This work was conducted in the framework of the DLR research project URBAN-Rescue and has received funding from the Clean Sky 2 Joint Undertaking (JU) under Grant Agreement No. 945583.

The JU receives support from the European Union's Horizon 2020 research and innovation programme and the Clean Sky 2 JU members other than the Union.

Armin Weiss

armin.weiss@dlr.de

1 Institute of Aerodynamics and Flow Technology, German Aerospace Center, DLR, Linder Höhe 51147, Cologne, Germany

2 Institute of Aerodynamics and Flow Technology, German Aerospace Center, DLR, Bunsenstraße 10, 37073 Göttingen, Germany

3 Institute for Aeroelastics, German Aerospace Center, DLR, Bunsenstraße 10, 37073 Göttingen, Germany

4 Institute of Aerodynamics and Gas Dynamics, University of Stuttgart, 70569 Stuttgart, Germany 


\section{Graphical abstract}
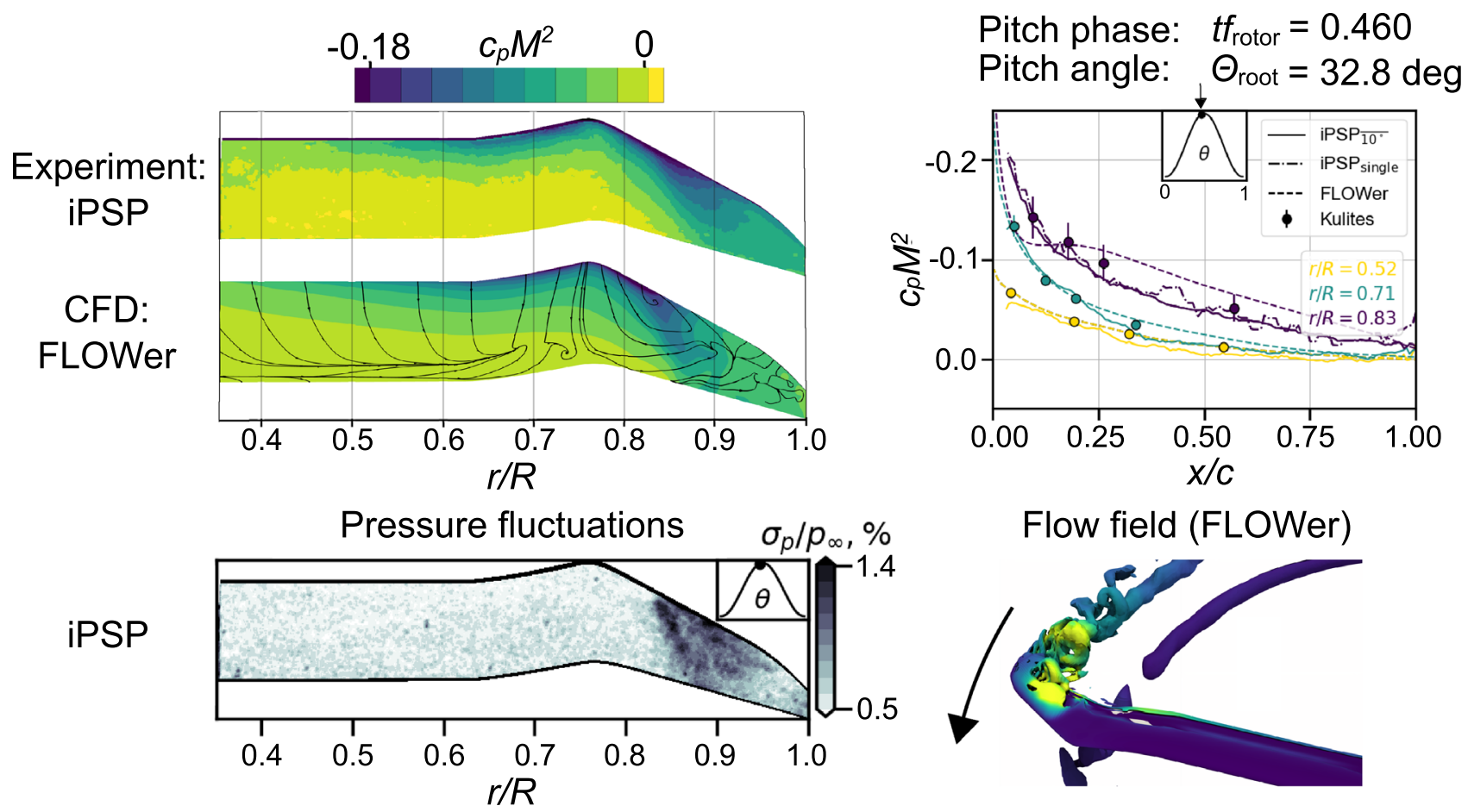

\section{Introduction}

Dynamic stall on helicopter rotor blades is of major interest in helicopter aerodynamics research as it causes impulsive and design-critical fluctuations of pitching moment, lift and drag (see Leishman 2006). The phenomenon occurs during forward flight and flight manoeuvre where the blade angle of attack is varied from low pitch angles on the advancing blade side to high pitch angles on the retreating blade side of the rotor disk. The flow topology is characterized by large coherent vortex structures evolving from the leading edge as the pitch angle increases, followed by their detachment and convection downstream.

The rotor test facility (RTG) of the German Aerospace Center (DLR) in Göttingen was developed by Schwermer et al. (2016) to study dynamic stall experimentally (Schwermer et al. 2019) and numerically (Goerttler et al. 2018; Letzgus et al. 2019) on rotor blades with a parabolic blade tip. More recently, double-swept rotor blades were developed for tests at the RTG (Müller et al. 2018). Unsteady Reynoldsaveraged Navier-Stokes (URANS) computations by Kaufmann et al. (2020) using DLR's TAU code (see Schwamborn et al. 2008) revealed insights into their dynamic stall behaviour and the associated flow topology.
In previous studies, dynamic stall was detected by pressure sensors (Bousman 1998; Schwermer et al. 2019) or tufts (Yamauchi et al. 1997; Schwermer et al. 2019). However, these techniques are limited in their spatial resolution and tuft images leave residual ambiguities in their interpretation. Gardner et al. (2016) used infrared thermography to optically detect dynamic stall on pitching airfoils and the technique was applied by Raffel et al. (2017) to deduce rotating blade stall maps that indicate geometrical regions on the blade where stalled flow occurs. Although the stall map presented in the latter study marked a breakthrough, the signal was rather weak and significant spatial averaging was needed to finally detect stalled flow regimes as a function of the blade radius and the pitch phase.

A powerful tool to analyse the global pressure footprint on rotor blades is the pressure-sensitive paint (PSP) technique. The technique is based on photokinetic interactions between the airflow and a luminescent coating applied on the object of investigation. When excited by light of a certain wavelength, the luminophores in the coating emit light at longer wavelengths. Both intensity and lifetime of the emitted light are reduced by a process called "oxygenquenching", which occurs due to diffused oxygen in the coating and depends on the local air pressure (see Liu et al. 
2021). PSP was used to study unsteady rotor aerodynamics of model (Disotell et al. 2014) and full-scale (Watkins et al. 2016) helicopters in forward flight. The studies were able to give insight into the unsteadiness of the flow field and yet were limited in their phase resolution, since the optical setup could only be directed at limited phase positions of the pitch cycle, which corresponds to a limited azimuthal range of the rotor disk in forward flight. A comprehensive review of the development and application of time-resolving pressure-sensitive paints on rotor blades and others was recently published by Peng and Liu (2019).

The application of PSP on rotor blades faces the challenge of acquiring sufficient luminescent output of the sensor at minimized exposure times of image sensors in order to avoid excessive rotational blur in the data. In the past, this challenge was tackled by the application of the "single-shot lifetime technique", where the luminescent decay is captured by two successively acquired images $\left(I_{\mathrm{G} 1}\right.$ and $I_{\mathrm{G} 2}$ ) after excitation of the luminophores by a single shot of laser light (see Gregory et al. 2009; Juliano et al. 2011). Residual image blur in the second image was usually unavoidable due to limitations of the double-shutter acquisition modes of the camera and the luminescent lifetimes of the PSP sensors used. Measures to overcome the problem of rotational blur were undertaken either by using complex rotating mirror setups (Raffel and Heineck 2014) or advanced post-processing techniques (Pandey and Gregory 2018), which usually hampered the signal-to-noise ratio (SNR) of the results. A different approach was presented by Weiss et al. (2017), who applied an advanced doubleshutter camera from Geisler (2017b) to PSP measurements on the parabolic tip rotor blades at the RTG only using collective-pitch settings. The camera inherently avoids image blur by limiting the exposure time of both successively acquired images to only a few microseconds.

The study presented here is first of all a follow-up to the work published by Weiss et al. (2017). Unlike in the 2017 campaign, the current study employs an improvement of the fast double-shutter camera introduced in 2017 and DLR's time-resolving PSP sensor to capture unsteady flow phenomena (abbreviated as "iPSP" sensor). In addition to the measurement technique development, iPSP is used for the first time in order to measure dynamic stall of the recently developed double-swept rotor blades in the RTG. The experimental work is complemented by numerical simulations performed at the Institute of Aerodynamics and Gas Dynamics at the University of Stuttgart using the flow solver FLOWer. The article comprises a characterization of the iPSP measurement system, followed by a description of the experimental and numerical setups. The findings are then presented for steady test cases and for time-resolved measurements to identify dynamic stall including a detailed comparison between experimental and numerical results.

\section{Experimental techniques and numerical simulations}

\subsection{Pressure-sensitive paint measurement system}

Surface pressures were measured using the single-shot lifetime technique. Pressures were obtained using the so-called "ratio-of-ratio" method ( $R o R$, as proposed by Ruyten and Sellers 2006), where ratio images of successively gated image intensities $\left(I_{\mathrm{G} 1}, I_{\mathrm{G} 2}\right)$ are processed according to Eq. 1 .

$$
\begin{aligned}
R o R & =\frac{I_{\mathrm{G} 1} / I_{\mathrm{G} 2}}{\left(I_{\mathrm{G} 1} / I_{\mathrm{G} 2}\right)_{\mathrm{ref}}}=\frac{\left(I_{\mathrm{G} 1} / I_{\mathrm{G} 2}\right)_{\mathrm{wind}-\mathrm{on}}}{\left(I_{\mathrm{G} 1} / I_{\mathrm{G} 2}\right)_{\mathrm{wind}-\mathrm{off}}} \\
& =\sum_{i, k=0}^{m, n=2} a_{i, k}\left(\frac{T}{T_{\mathrm{ref}}}\right)^{i}\left(\frac{p}{p_{\text {ref }}}\right)^{k}
\end{aligned}
$$

The RoR method implies that the image ratio at the condition of interest (wind-on) is divided by the image ratio at a reference condition (ref or wind-off), where pressure $p_{\text {ref }}$ and temperature $T_{\text {ref }}$ of the iPSP sensor are homogeneous and known. In Eq. 1, the polynomial coefficients $a_{i k}$ were obtained from a calibration measurement. The second order polynomial was chosen as it proved to yield a good approximation for the nonlinear Stern-Vollmer relation (see Liu et al. 2021) in the preceding study by Weiss et al. (2017). Moreover, the pressure distribution $p$ can only be obtained if the temperature distribution $T$ is known.

The employed iPSP sensor is the result of a collaborative work between DLR and the Münster University of Applied Sciences (Ondrus 2020) and states an improvement of the sensor. It is based on the work from Scroggin et al. (1999) and Gregory et al. (2002). The iPSP sensor used was platinum tetra(pentafluorophenyl)porphyrin (PtTFPP Puklin et al. 2000) as the luminophore in the active layer, which was dissolved in toluene. The active layer was spray coated onto a base layer (also spray coated) consisting of ceramic particles (titanium silicon oxide $\mathrm{TiSiO}_{4}, 50 \mathrm{~nm}$ ) embedded in a polymer matrix (Duromax B-1000), a dispersant (Aldrich PANa 4160029) and distilled water. A schematic sketch of the layer setup is provided in Fig. 1a. Microscopic images of the paint surface were acquired by a field emission scanning electron microscope (JSM-6700F, JEOL) by Egami (2020). An acquired top view image is displayed in Fig. $1 b$. It shows the surface structure, which is principally formed by the polymer matrix and the embedded $\mathrm{TiSiO}_{4}$ particles. Note that the scale does not allow to distinguish the components listed in the schematic sketch in Fig. 1b.

The coating thickness and surface roughness were determined by a laser microscope (VK-X200, Keyence) to be $h=28.2 \mu \mathrm{m}$ and $R_{a}=9.8 \mu \mathrm{m}$, respectively (Egami 2020). It is believed that a turbulent boundary layer is not affected by surface roughness unless the roughness elements protrude 
a

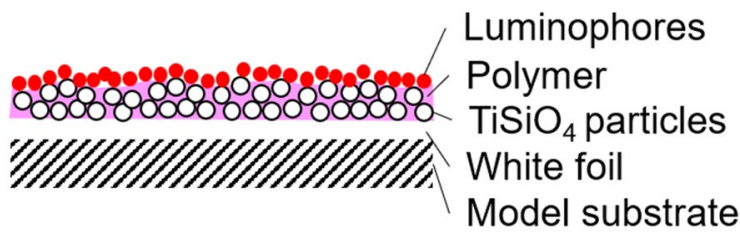

b

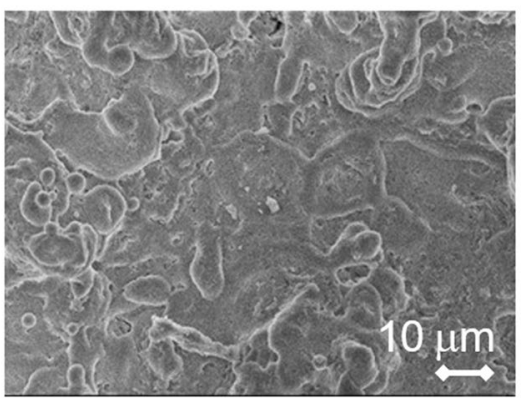

Fig. 1 a Schematic sketch of iPSP sensor coating. b Microscopic top view of the iPSP sensor from Egami (2020)

the viscous sublayer (in normalized units) at $y^{+}=5$ (see Schlichting and Gersten 2017). A detailed investigation of the intrusiveness of the iPSP coating was beyond the scope of this study, especially because pressure transducers were mounted on a different blade than the one coated with iPSP. Following the procedure from Schairer et al. (1998) and assuming the absolute roughness height of $R_{a}$, a unit Reynolds number equivalent to $\mathrm{Re}_{\text {tip }}=5.95 \times 10^{5}$ and Schlichting's skin friction law for a turbulent flat plate, the calculated non-dimensional boundary-layer thickness stays below $y^{+}<2.3$. The iPSP coating is thus considered to be "hydraulically smooth" and non-intrusive. However, Schairer et al. (1998) mentioned that even nominally "hydraulically smooth" PSP coatings can possibly affect, e.g. the stall angle.

The time response of the sensor was previously characterized by Sugimoto et al. (2017) and presented by Gößling et al. (2020), where a $-3 \mathrm{~dB}$ frequency limit of about $6 \mathrm{kHz}$ was identified. In this campaign, the tested rotor frequency was $23.6 \mathrm{~Hz}$ which yields a time response equivalent to 1.4 deg azimuth. The iPSP and base layer were coated onto a white foil ( $3 M$ Wrap Film Series 1090, $\approx 90 \mu \mathrm{m}$ ), which was wrapped around the black rotor blade (made out of carbon fibre-reinforced plastic, see Sect. 2.2) in order to increase the luminescent output of the paint and to protect the blade surface finish from aggressive solvents.

The iPSP was excited at a wavelength of $532 \mathrm{~nm}$ by a laser system consisting of two neodymium-doped yttriumaluminum-garnet (Nd:YAG) lasers (Quantel Evergreen 200).

The iPSP-signal was recorded by the nominally four megapixel frame-optimized exposure FoxCam $4 M$ camera (see Geisler 2014, 2017a). The optimized double-shutter was previously used as essential part of the single-shot lifetime measurement system by Weiss et al. (2017), as it allowed image acquisition of two frames in direct succession with exposures of both frames limited to only a few microseconds. The image acquisition mode used by Weiss et al. (2017) is referred to as "Mode A" here and schematically sketched in Fig. 2 (top).

It used only the even rows of the charge-coupled device (CCD) sensor (Geisler 2017b) to successively acquire frames during gates $G 1$ and $G 2$. Odd sensor rows remained unused in order to realize the described double-shutter acquisition with limited exposure time for $G 2$. In this campaign, a different acquisition mode was used. It is further referred to as "Mode B" and was described in detail as "FOX mode with two short exposure times" in Figs. 2 and 3b (see Geisler 2017a). The acquisition scheme for Mode B is also shown in Fig. 2 (bottom). The advantage of Mode B as compared to Mode A is that every sensor line is exploited, which doubles the luminescence signal in the image and thus the sensitivity when compared to Mode A. The duration of $G 1$ is equal to the trigger pulse length $\tau$. After the end of $G 1$, charges are transferred to the vertical register and charges of two

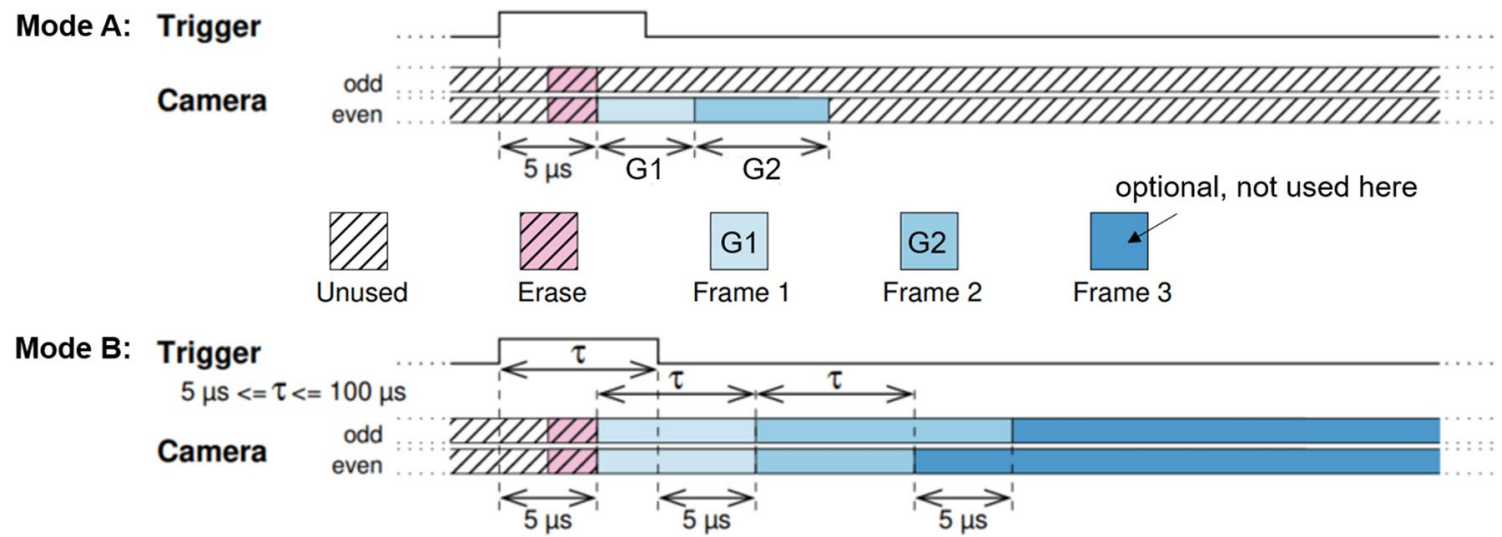

Fig. 2 Image acquisition schemes of FoxCam4M: "Mode A" used by Weiss et al. (2017), "Mode B" used in this campaign 

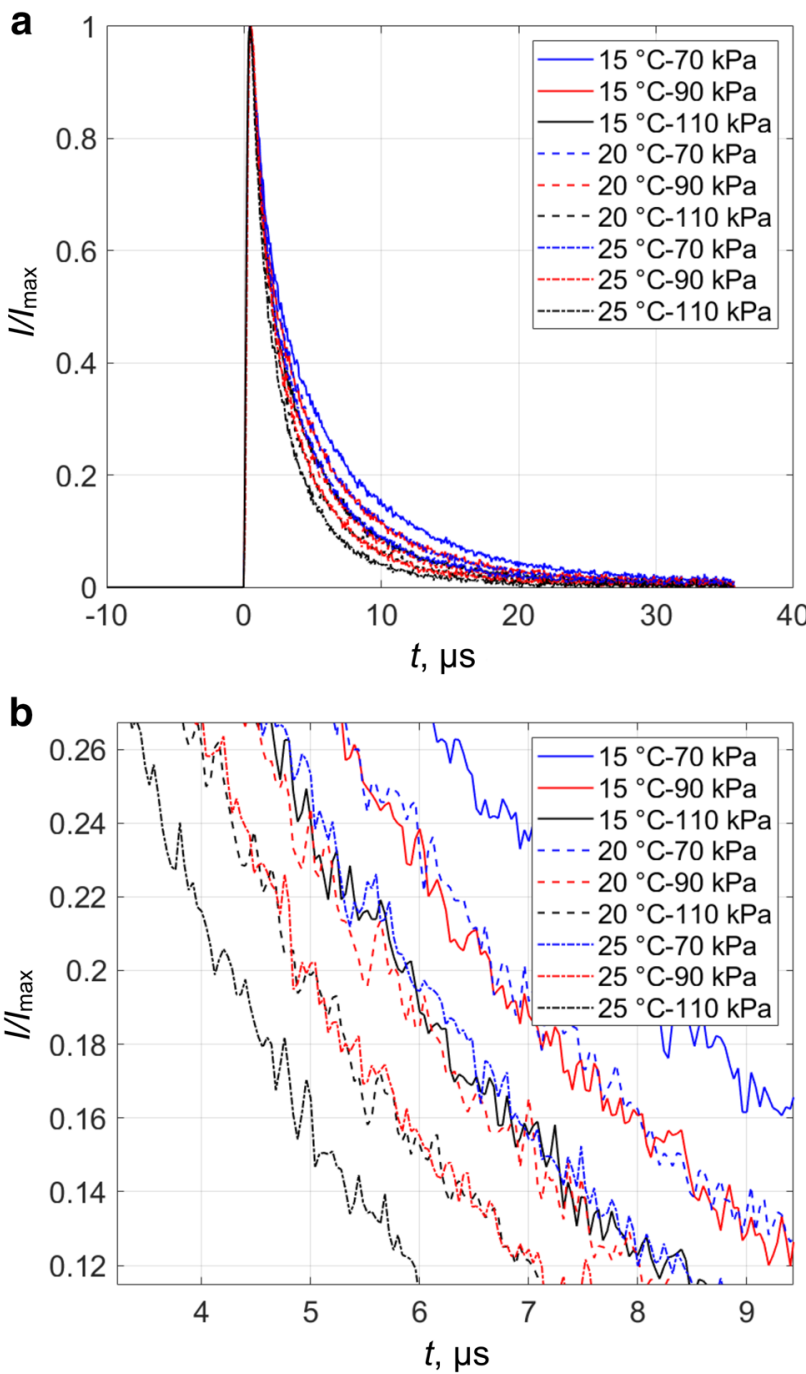

Fig. 3 Luminescence decay curves for the iPSP sensor

neighbouring pixels from odd and even lines are added. Due to technical reasons, the exposure is $G 2=\tau$ for even lines and $G 2=\tau+5 \mu$ s for odd lines, with $5 \mu$ s $\leq \tau \leq 100 \mu$ s. During the extra $5 \mu$ s for odd sensor lines, charges of even lines are transferred to the vertical register, the vertical register is shifted and finally the charges of odd lines are added (see Geisler 2017a, for more detail).

Considering Mode B in this campaign, the maximum allowable image blur occurs in $G 2$ and is limited by the duration of $\tau+5 \mu$ s. The selection of $\tau=10 \mu$ s yields a blur of $<1.5 \mathrm{~mm}$ at the blade tip at $f_{\text {rotor }}=23.6 \mathrm{~Hz}$, which corresponds to less than $2 \%$ of the chord at a blade tip speed of $96.7 \mathrm{~m} / \mathrm{s}$.

In a pretest, the luminescent decay of the iPSP sensor was recorded in order to determine the timing between the laser pulse and the beginning of $G 1$. The decay curves were recorded by a photo-multiplier tube at $25 \mathrm{MHz}$ as

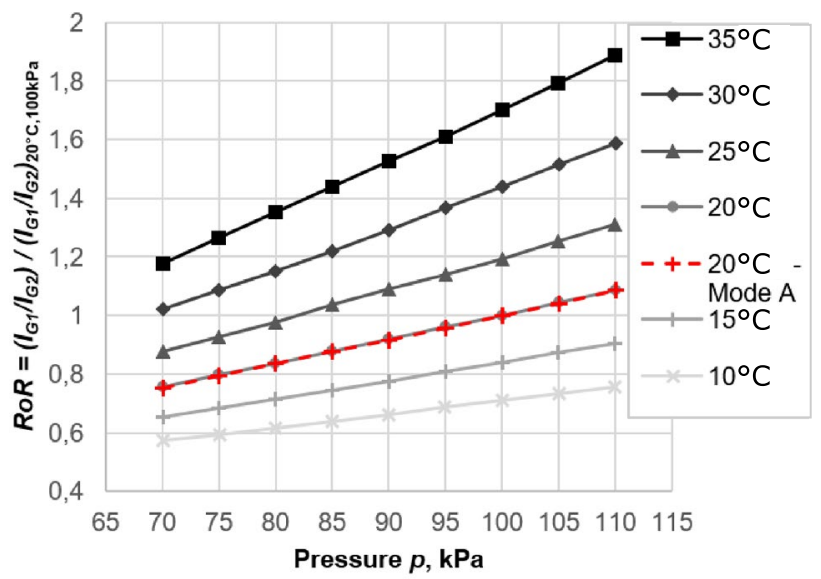

Fig. 4 Calibration curves for the iPSP sensor: ratio of ratios RoR as a function of pressure for different temperatures, using Mode B at $\tau=10 \mu \mathrm{s}$ and $\Delta t_{\mathrm{Puls}-\mathrm{G} 1}=+8 \mu \mathrm{s}$

the sensor was excited by a green LED pulse (Hardsoft Illuminator $I L 106 G$ ) with a peak emission wavelength of $528 \mathrm{~nm}$. In order to separate the excitation light from the iPSP signal, a $650 \pm 50 \mathrm{~nm}$ bandpass filter was used in front of the photo-multiplier tube and a $520 \pm 18 \mathrm{~nm}$ bandpass filter was used in front of the LED. Yorita et al. (2018b) recently showed that the PSP luminescence decay time depends on the excitation pulse width. In order to simulate the excitation by a laser pulse $(\approx 20 \mathrm{~ns})$, the LED pulse was therefore limited to the minimum adjustable pulse width of $0.2 \mu$ s and powered by $250 \mathrm{~A}$. The luminescence decay curves are presented in Fig. $3 \mathrm{a}$ as normalized intensity values versus time with a zoom into the graph in part $b$ of the figure. The displayed graphs show the expected faster luminescence decay at increased pressures and temperatures.

The luminescence decay curves were used to set the timing between the laser and camera in the wind tunnel test. A delay of $\Delta t_{\text {Puls-G1 }}=+8 \mu$ s was chosen between the start of $G 1$ and the laser pulse; i.e. $G 1$ starts $8 \mu$ s prior to the laser. The criteria for this choice were similar to the ones in the preceding study (Weiss et al. 2017).

The iPSP system was calibrated in a dedicated test using the laser for excitation of a coated iPSP sensor sample and the FoxCam $4 M$ for image acquisition using the previously described laser and camera settings. Image intensities were evaluated as an averaged value of a $40 \times 40$ pixel region of interest and the result is presented in Fig. 4, where the $R o R$ values according to Eq. 1 are plotted against pressure for different temperatures.

The resulting pressure- and temperature sensitivities $s_{p}$ and $s_{T}$ at $p=100 \mathrm{kPa}$ and $T=20^{\circ} \mathrm{C}$ are calculated as follows: 


$$
\begin{aligned}
s_{p, 100} \mathrm{kPa}, 20^{\circ} \mathrm{C} & =\frac{\operatorname{RoR}_{1} 05 \mathrm{kPa}-R_{0} R_{95} \mathrm{kPa}}{10 \mathrm{kPa}} * 100 * 100 \\
& =84.5 \frac{\%}{100 \mathrm{kPa}},
\end{aligned}
$$

$s_{T}, 100 \mathrm{kPa}, 20^{\circ} \mathrm{C}=\frac{\operatorname{RoR}_{25^{\circ} \mathrm{C}}-R_{o} R_{15^{\circ} \mathrm{C}}}{10 \mathrm{~K}}=3.6 \frac{\%}{K}$.

At $20^{\circ} \mathrm{C}$, the calibration curve for the camera acquisition Mode $\mathrm{A}$ is added. This mode was used in the 2017 campaign and it was used again in this study for comparison at steady test cases. Mode A and Mode B are best compared when choosing $G 1=10 \mu$ s and $G 2=12.5 \mu$ s for Mode A. Since for Mode B, where $G 2_{\text {even }}=10 \mu$ s for even sensor lines and $G 2_{\text {odd }}=15 \mu$ s for odd sensor lines, differences of the image ratios $I_{\mathrm{G} 1} / I_{\mathrm{G} 2}$ between the two modes occur only due to the non-linearity of the exponential luminescence decay during the extra $5 \mu$ s the odd sensor lines are exposed during $G 2$ of Mode B. In Fig. 4, the calibration curves for both modes at $20^{\circ} \mathrm{C}$ are very similar. Hence, this non-linearity is insignificantly small, which is due to the exponential decay of the fluorescence lifetime.

\subsection{Wind tunnel setup and test conditions}

The experiment was conducted in the rotor test facility of DLR in Göttingen (RTG). An image and schematic view of the measurement setup are provided in Figs. 5 and 6, respectively. The four-bladed rotor was placed $2.3 \mathrm{~m}$ in front of the nozzle outlet of the Eiffel-type wind tunnel. The rotor axis was perpendicular to the nozzle outlet plane which had a cross section of $1.6 \mathrm{~m} \times 3.4 \mathrm{~m}$ (height $\times$ width). The wind

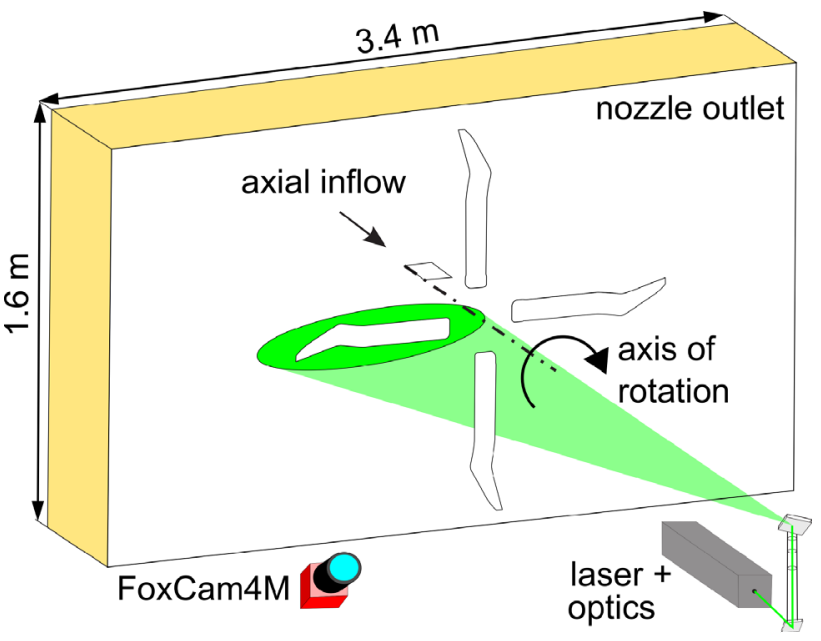

Fig. 6 Schematic sketch of the measurement setup for iPSP test of four-bladed double-swept rotor at the RTG

tunnel provided an axial inflow of $v_{\infty}=2 \mathrm{~m} / \mathrm{s}$ in order to prevent blade-vortex interaction and the recirculation of blade tip vortices.

The Mach-scaled and double-swept rotor blades were designed by Müller et al. (2018). They had a tip radius of $R=0.652 \mathrm{~m}$ and a chord length of $c=0.072 \mathrm{~m}$ and comprised a blended geometry of EDI-M109 and EDIM112 airfoils. More details, e.g. regarding the sweep and twist distribution are provided by Müller et al. (2018). The reduced frequency (based on the semi chord-length) at $75 \%$ of the blade tip corresponds to $k_{75}=0.074$. One of the blades was equipped with sealed gauge unsteady pressure transducers (Kulite LQ-062) at different radial positions

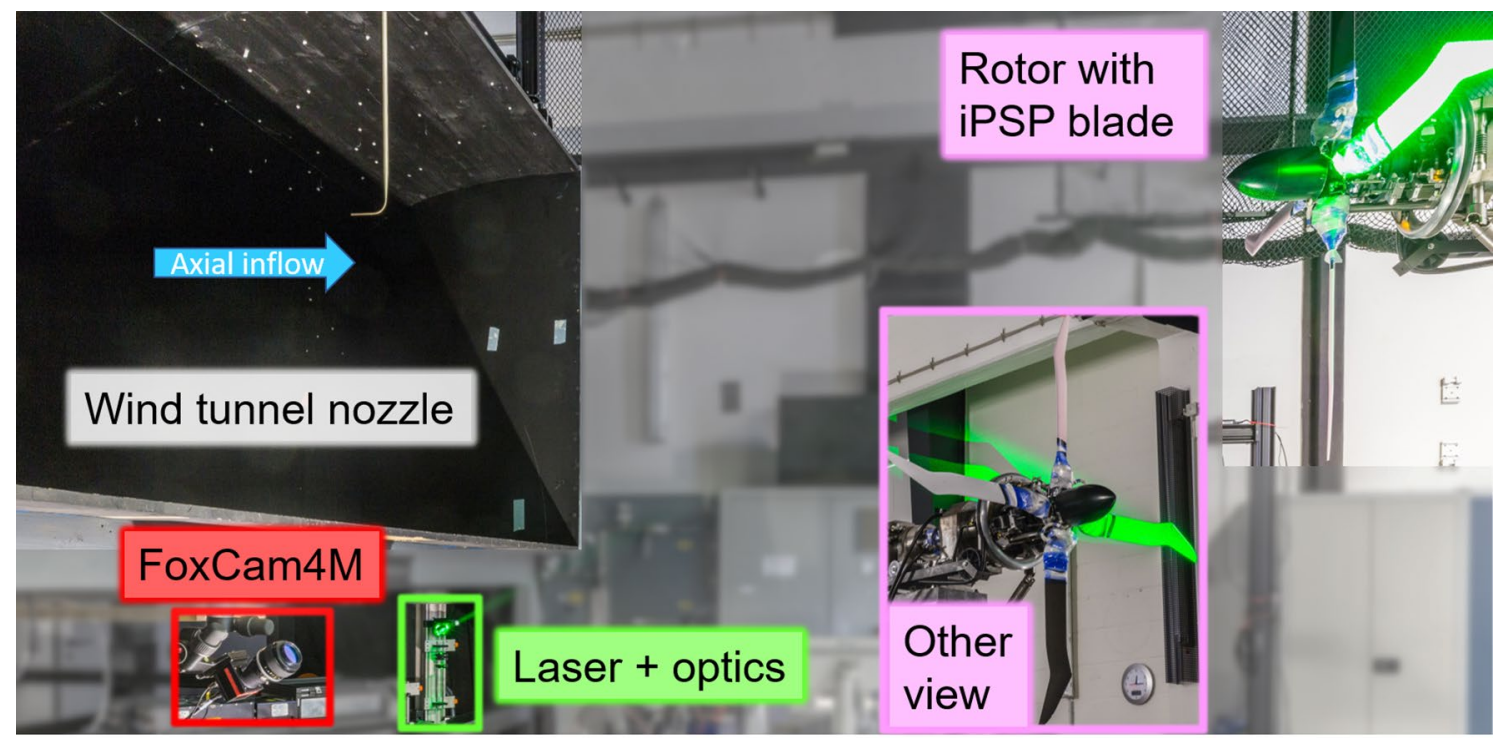

Fig. 5 Image of the measurement setup for iPSP test of four-bladed double-swept rotor at the RTG 
Fig. 7 Planform of doubleswept rotor blade and positioning of instrumentation

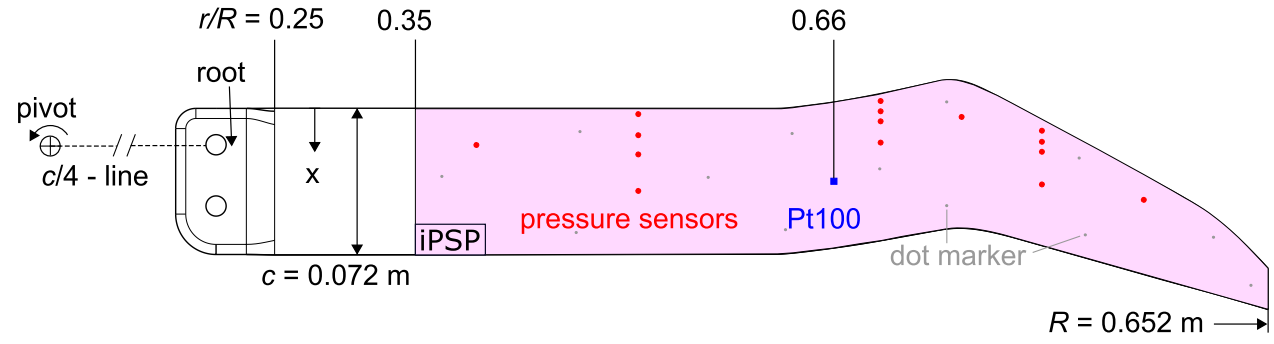

\begin{tabular}{|c|c|c|c|c|c|c|}
\hline$r / R$ & 0.40 & 0.52 & 0.71 & 0.77 & 0.83 & 0.90 \\
\hline \multirow[t]{4}{*}{$x / c$} & 0.251 & 0.042 & 0.049 & 0.251 & 0.094 & 0.251 \\
\hline & & 0.192 & 0.124 & & 0.178 & \\
\hline & & 0.321 & 0.195 & & 0.261 & \\
\hline & & 0.545 & 0.337 & & 0.571 & \\
\hline
\end{tabular}

Table 1 Positioning of pressure transducers on the rotor blade
Table 2 Test conditions

\begin{tabular}{lllll}
\hline$f_{\text {rotor }}=23.6 \mathrm{~Hz} ; v_{\infty}=2.0 \mathrm{~m} / \mathrm{s}$ & & & \\
\hline Cyclic test cases & $\bar{\Theta}_{\text {root }}, \mathrm{deg}$ & $\hat{\Theta}, \mathrm{deg}$ & $\mathrm{M}_{\text {tip }}$ & $\mathrm{Re}_{\text {tip }}, 10^{5}$ \\
\hline $\mathrm{C} 1$ & 27 & 6 & 0.282 & 5.84 \\
$\mathrm{C} 2$ & 32 & 6 & 0.282 & 5.84 \\
Collective polar & & & \\
$\bar{\Theta}_{\text {root }}=6-38 \mathrm{deg} ; \Delta \bar{\Theta}_{\text {root }}=1 \mathrm{deg}$ & $0.284-0.285$ & $5.92-5.95$ \\
\hline
\end{tabular}

et al. 2019). Table 2 lists the test conditions of the different runs tested during the campaign.

The laser used for iPSP excitation was installed underneath the bottom corner of the wind tunnel nozzle. An optical setup of mirrors and lenses redirected the laser beam towards the rotor reference position and created an elliptic spot in order to illuminate the coated blade area, as depicted in Fig. 5. Due to the enhanced acquisition mode of the FoxCam4M, a $400 \mathrm{~mJ} /$ pulse laser system of two cavities $(2 \times 200 \mathrm{~mJ} /$ pulse $)$ yielded sufficient luminescent output of the iPSP. In contrast, a $800 \mathrm{~mJ} /$ pulse laser system was used in the previous campaign in 2017 for excitation of the PSP to acquire sufficient signal with the previously used acquisition Mode A. The pulses of both lasers were aligned and emitted in direct succession within 50 ns. The FoxCam $4 M$ was mounted underneath the nozzle outlet and directed towards the centre of the iPSP-coated blade area in the reference position at a distance of about $2.5 \mathrm{~m}$. A $85 \mathrm{~mm}$ Nikon Nikkor lens was used with an aperture opening of $\mathrm{f} / 2.8$. The viewing angle of $\approx 23 \mathrm{deg}$ corresponded roughly to the mean of tested pitch angles and thus allowed for omitting the use of a lens adapter to fulfil the Scheimpflug condition (Bickel et al. 1985). With this setup, a resolution of $2.2 \mathrm{px} / \mathrm{mm}$ 
was achieved (after application of the $2 \times 1$ binning, as described in Sect. 2.4.1).

\subsection{Data acquisition}

The iPSP data acquisition in principle followed the procedure as outlined in Weiss et al. (2017). Therefore, only a brief description is given here, and differences are pointed out. For each data point, a set of 128 dark and reference wind-off double frames, i.e. $I_{\mathrm{G} 1}$ and $I_{\mathrm{G} 2}$ images, were acquired. In contrast to the campaign described by Weiss et al. (2017) more time (at least $15 \mathrm{~min}$ ) between starting the rotor and acquiring images in the wind-on condition was spent for the blades to reach equilibrium temperature. At $f_{\text {rotor }}=23.6 \mathrm{~Hz}$, images were acquired every second revolution at $f_{\text {acq }}=11.8 \mathrm{~Hz}$. The image acquisition rate was limited by both the laser and camera, which allowed maximum data acquisition rates of $f_{\text {max.laser }}=15 \mathrm{~Hz}$ and $f_{\text {max,camera }}=20 \mathrm{~Hz}$ (for double frame acquisition), respectively. For test cases with the collective blade setting only, a series of 128 double frames was acquired in wind-on condition. For the unsteady test cases with cyclic-pitch settings, the speed of the swash plate rotation was adopted such that 1600 double frames were recorded during the entire pitch cycle, yielding a phase resolution of $\Delta t f_{\text {rotor }}=1 / 1600$, i.e. a 0.225 deg azimuth, and an acquisition time of about $136 \mathrm{~s}$.

Data from pressure transducers and $P t 100$ are recorded by an acquisition system with a $-3 \mathrm{~dB}$ cutoff frequency of $19 \mathrm{kHz}$. For collective test cases, all rotor data were averaged over at least $10 \mathrm{~s}$. More detailed information about the rotor data acquisition is provided by Schwermer et al. (2016, 2019).

\subsection{Data processing}

\subsection{1 iPSP data reduction}

After image read out, a $2 \times 1$ binning was applied to increase the SNR by a factor of $\sqrt{2}$ and to restore the original image aspect ratio. 2D- and 3D-image processing were performed using the in-house developed software package ToPas (as in Klein et al. 2005), involving the following steps:

$2 D$ image processing:

- Subtraction of dark signals from all images,

- Application of a flatfield correction to all raw images in order to remove CCD-characteristic stripe pattern as similarly observed in Fig. 16a of Weiss et al. (2017),

- Wind-off ratio $\bar{R}_{\text {wind-off }}={\overline{\left(I_{\mathrm{G} 1} / I_{\mathrm{G} 2}\right)}}_{\text {wind-off }}$ : Ratio calculation and ensemble averaging of 128 ratio images,

- Wind-on single-shot ratio $R_{\text {wind-on }}=\left(I_{\mathrm{G} 1} / I_{\mathrm{G} 2}\right)_{\text {wind-on }}$ : Image registration of all $G 2$ images to the respective $G 1$ image before division. All ratio images were aligned to the averaged first frame in the wind-off condition. The alignment procedure accounts for rotation and translation using thirteen dot markers applied to the coated surface,

- Ratio-of-ratio calculation: Collective test cases with an ensemble average of 128 wind-on single-shot ratios: $\overline{R o R}=\bar{R}_{\text {wind-on }} / \bar{R}_{\text {wind-off }}$, Cyclic test cases with singleshot wind-on ratios: $R o R=R_{\text {wind-on }} / \bar{R}_{\text {wind-off }}$.

\section{$3 D$ image processing:}

- Image projection of $R o R$ results on a 3D grid with a resolution of 0.95 nodes/px; for this, the 3D positions of the dot markers were measured beforehand using a tactile measurement probe; the projected images were $7 \times 7$ median-filtered. The filter eventually removed residual noise and the small blank spaces at the dot marker locations by assigning the median value of the respective kernel to each pixel before mapping was applied,

- Application of calibration polynomial (Eq. 1) using the temperature correction methodology as detailed by Weiss et al. (2017) and briefly discussed in Sect. 2.4.2,

- Offset correction using data from pressure sensors, as described in Sect. 2.4.3,

- Masking of area close to the leading edge where no data were available due to shading.

\subsubsection{Temperature correction}

The applied temperature correction methodology follows the procedure of Weiss et al. (2017) and considers the one-dimensional adiabatic wall temperature distribution $T_{\text {aw }}$ according to Eq. 5, where $T_{\infty}$ is the free stream temperature, $F_{\text {rec }}=0.85$ is the recovery factor of turbulent boundary layers, $\kappa=1.4$ is the ratio of specific heats and $R_{\text {air }}=287 \mathrm{~J}=(\mathrm{kgK})$ is the ideal gas constant for air. $T_{\text {aw }}$ varies quadratically along the blade radius, while it is anchored by the $P t 100$ reading at $r / R=0.66$ (see Fig. 8).

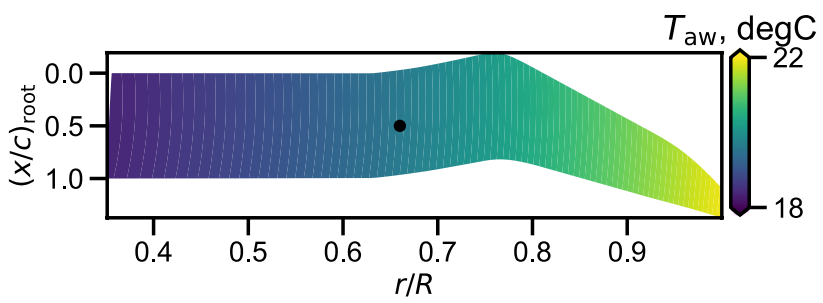

Fig. 8 Exemplary temperature map used for temperature correction of iPSP data and corresponding to adiabatic wall temperature according to Eq. 5; the black dot marks the position of the Pt100 sensor 
$T_{\text {aw }}=T_{\infty}+F_{\text {rec }} \cdot \frac{\left(\frac{r}{R} v_{\text {tip }}\right)^{2}}{2\left(\kappa R_{\text {air }}\right)(\kappa-1)}$

The method was previously applied by Disotell et al. (2014) and Watkins et al. (2016). It ignores adiabatic wall temperature variations along the chord, which depend on the local velocity distribution and therefore on the local pressure distribution (Jiao et al. 2020). However, the pressure distribution in this study is not known apriori. Considering that Weiss et al. (2017) achieved very good agreement between PSP and pressure transducer data along the chordwise direction $(<250 \mathrm{~Pa})$, the same approach was considered here.

It should also be mentioned that in the current study, a single temperature map is used to correct the unsteady pressure distributions measured during the pitch cycle. This is justified because the temperature variation across the pitch cycle is expected to be negligible, mainly because the total temperature was constant during the pitch cycle in the RTG setup.

\subsubsection{Offset correction}

PSP results from wind tunnel measurements oftentimes yield biased results when compared to conventional pressure transducer results. They can be due to uncontrollable test environments but the primary reason is the uncertainty of the surface bulk temperature, for instance at reference condition (see McLachlan and Bell 1995). A possible way to correct this bias is an in situ calibration of the PSP, where luminescence intensities on the model are correlated with appropriate reference measurements within a significant range (see e.g. Yorita et al. 2018a). Alternatively, an offset correction of the results can be applied by "anchoring" the iPSP data with pressure transducer readings at the same location. In this study, the latter approach was considered. The applied offset was obtained from the mean of the differences between time-averaged data of unsteady pressure transducers and iPSP values at the same location. Recall that iPSP was coated on a different but nominally identical blade than the one instrumented with unsteady pressure transducers in order to avoid a temperature bias in iPSP data due to heating by the unsteady pressure transducers (as in Weiss et al. 2017). The procedure was applied in the same manner for both collective-pitch and cyclic-pitch cases and leaves the calculation of pressure fluctuations from iPSP results unaffected.

\subsubsection{Measurement uncertainty}

Even though the expected differences of the calculated adiabatic wall temperature to the real temperature distribution on the blade surface are expected to be small, the pressure error due to uncorrected or faulty assumed temperatures of the iPSP sensor in this study is $4.26 \mathrm{kPa} / \mathrm{K}$ at $20^{\circ} \mathrm{C}$ and 100 $\mathrm{kPa}$.

iPSP results were compared to fast-response pressure transducer readings for all collective data points and at thirteen different positions as listed in Table 1, except for the ones closest to the leading edge at $[x / c, r / R]=[0.042,0.52]$ and $[0.049,0.71]$. These locations were shaded for some data points, and therefore, iPSP data are partially unavailable here. Recall, that iPSP and pressure transducer data are sampled on different and yet structurally identical blades. The resulting mean of all absolute deltas was $\approx 750 \mathrm{~Pa}$. This mean difference is higher than the $\approx 250 \mathrm{~Pa}$ provided in the preceding study (Weiss et al. 2017). This is probably due to the fact that in the 2017 campaign the deltas were evaluated at a single radial position only, where both surface temperatures and PSP results were anchored. In this study however, the difference value is based upon pressure transducer readings across $0.52 \leq r / R \leq 0.90$.

\subsection{Numerical simulations}

The high-fidelity computational fluid dynamics (CFD) simulations used for comparison with the test case $\mathrm{C} 1$ with the cyclic-pitch setting were conducted at the Institute of Aerodynamics and Gas Dynamics of the University of Stuttgart using the block-structured finite-volume flow solver FLOWer (Raddatz and Fassbender 2005). In a previous simulation of the RTG, the influence of the test rig was found to be negligible (Letzgus et al. 2019). Thus, only isolated rotor blades were modelled. A uniform axial inflow of $2.2 \mathrm{~m} / \mathrm{s}$ was used to simulate the wind-tunnel inflow in the experiment (measured at $2.0 \mathrm{~m} / \mathrm{s}$ ). The $\mathrm{O}$-type rotor blade grids contained about 11.5 million grid cells each and were embedded into a Cartesian off-body grid with an additional 10.5 million cells using the Chimera technique. For spatial and temporal discretization, the second-order central-differences Jameson-Schmidt-Turkel scheme and a second-order implicit dual-time-stepping method with a physical time-step size corresponding to $0.125 \mathrm{deg}$ azimuth was applied, respectively. While the flow was considered to be fully turbulent at all times, a delayed detached-eddy simulation approach with Menter-SST (shear stress transport, see Gritskevich et al. 2013) as the underlying RANS turbulence model was carried out. Furthermore, a well-established (Letzgus et al. 2020) weak fluid-structure coupling between FLOWer and the computational structural dynamics (CSD) code CAMRAD II (Johnson 1998) was used. For this, the rotor blade was modelled as an Euler-Bernoulli beam to capture the elastic deformation.

Kaufmann et al. (2020) performed URANS computations using the RTG test setup and DLR's TAU code with 
an isolated four-bladed rotor configuration and the same double-swept blade geometry as in this study. Their test case TC 3 was set to conditions corresponding to the test case C2 in this study, except for the axial inflow, which was also set to $2.2 \mathrm{~m} / \mathrm{s}$ as the simulation of test case $\mathrm{C} 1$ with FLOWer. Details of the numerical setup and technique are provided in Kaufmann et al. (2020).

\section{Results}

\subsection{Collective-pitch cases}

\subsubsection{Camera acquisition mode comparison}

In Fig. 9, the surface pressure maps of single-shot results at $\bar{\Theta}_{\text {root }}=22 \mathrm{deg}$ are shown for camera acquisition Mode A and Mode B, respectively. The surface pressures are displayed as $c_{p} M^{2}$ according to Eq. 6 .

$c_{p} M^{2}=\frac{p-p_{\infty}}{\rho_{\infty} / 2 \cdot a_{\infty}^{2}}$

In this expression, $\rho_{\infty}$ is the air density of the axial inflow and the pressure coefficient $c_{p}$ and the Mach number $M$ are defined as

$c_{p}=\frac{p-p_{\infty}}{\frac{\rho_{\infty}}{2} u^{2}} ; \quad M=\frac{u}{a_{\infty}}$

with $u$ as the rotation speed at the respective radial position and $a_{\infty}$ as the speed of sound of the axial inflow. As expected from the description of the different modes in Sect. 2.1, the enhanced Mode B yields a result with a better SNR (factor of $\sqrt{2}$ ), since the signal is doubled while maintaining the same noise level as compared to the Mode A result. On the other hand, the qualitative pressure distribution is the same
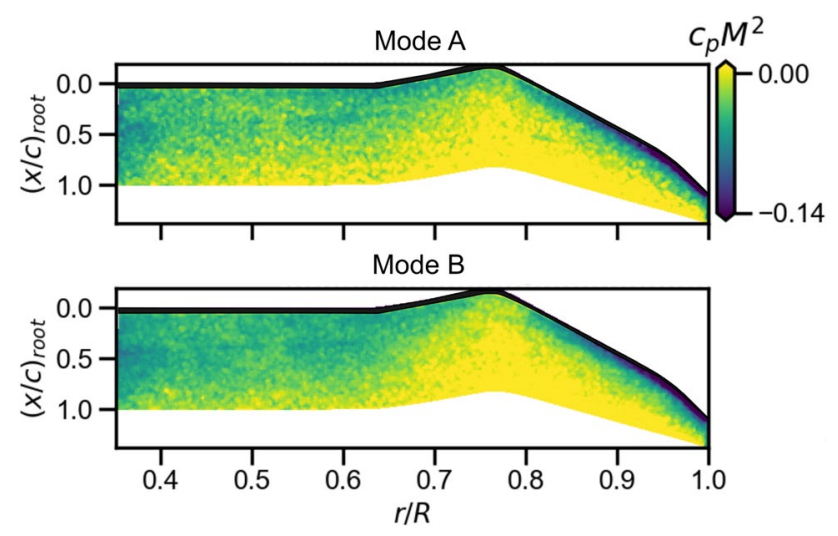

Fig. 9 Pressure maps from single-shot results using camera acquisition Mode A (top) and the improved Mode B at $\bar{\Theta}_{\text {root }}=22 \mathrm{deg}$ for both results. Note especially the pronounced suction peak close to the blade leading edge outboard of the blade apex. All further results presented below were acquired using the acquisition Mode B.

\subsubsection{Collective-pitch series}

A series of surface pressure maps for different collective root pitch angles is presented in Fig. 10a-d. The results all comprise a larger SNR than the single-shot results in Fig. 9 (by a factor of $\sqrt{128}$ ) due to ensemble averaging of 128 samples. At $\bar{\Theta}_{\text {root }}=24 \mathrm{deg}$ and $25 \mathrm{deg}$ the surface pressure maps indicate that the suction peaks outboard and inboard of the blade apex are more pronounced than at the apex itself at $r / R \approx 0.77$. At $\bar{\Theta}_{\text {root }}=25 \mathrm{deg}$, an oblique region with low pressures evolves close to the blade tip between $0.95<r / R<1$. As detailed in Sect. 3.2, this region of comparatively low pressures results from the footprint of the blade tip vortex, which starts to separate from the leading edge and close to the blade tip at $\bar{\Theta}_{\text {root }} \approx 25 \mathrm{deg}$. As the root pitch angle increases, the vortex becomes stronger, and separation starts further inboard, resulting in a larger oblique region with lower pressures at $\bar{\Theta}_{\text {root }}=33 \mathrm{deg}$ and between $0.85<r / R<0.93$. Note also the pronounced suction peak at $\bar{\Theta}_{\text {root }}=33$ deg between $0.65<r / R<0.77$ which diminishes as the root pitch angle is further increased to $\bar{\Theta}_{\text {root }}=36 \mathrm{deg}$, indicating stalled flow inboard of the blade apex.

The findings are supported by the corresponding tangential cuts at $r / R=0.71$ and $r / R=0.83$ which are plotted against the streamwise coordinate $x / c$ on the right-hand side of the figure with a comparison between results from iPSP and pressure transducers. The indicated errorbar for iPSP in Fig.10e corresponds to twice the mean absolute difference between results from unsteady pressure transducers and iPSP, i.e. $\pm 750 \mathrm{~Pa}$, as described in Sect. 2.4.4. The streamwise coordinate starts at the blade leading edge and is normalized with the chord length at the respective blade radius. In each of the displayed $c_{p} M^{2}$ plots the qualitative streamwise pressure distribution indicated by the pressure transducers is matched and complemented by the iPSP results. The cycle-to-cycle standard deviations of the pressure transducer readings, denoted as $\sigma c_{p}$, are indicated by bars. At $\overline{\boldsymbol{\Theta}}_{\text {root }}=33 \mathrm{deg}$ the $\sigma c_{p}$ readings in Fig. $10 \mathrm{~g}$ indicate increased fluctuations especially close to the leading edge at $r / R=0.83$ as compared to $r / R=0.71$ that are induced by the shedding leading-edge vortex outboard of the apex. These fluctuations are further increased at $\bar{\Theta}_{\text {root }}=36 \mathrm{deg}$. Here, increased fluctuations also occur at $r / R=0.71$, and the pressure distribution is flat, indicating stalled flow. On the other hand, at $r / R=0.83$ the pressure distribution still comprises a significant slope with lower pressures close to the leading edge induced by the shedding vortex. 

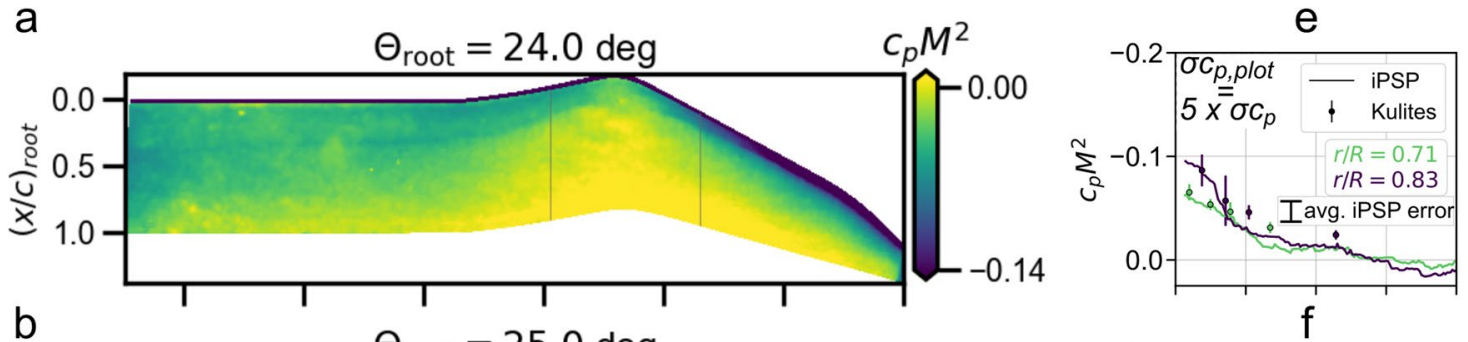

b

$\Theta_{\text {root }}=25.0 \mathrm{deg}$
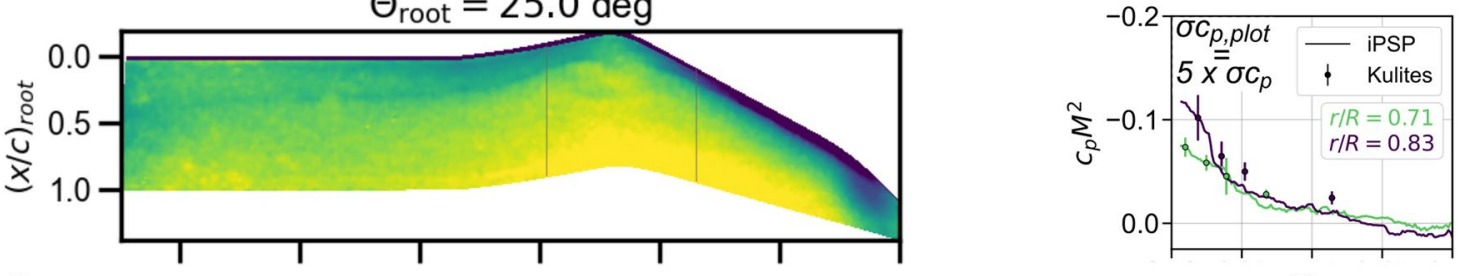

C

$\Theta_{\text {root }}=33.0 \mathrm{deg}$
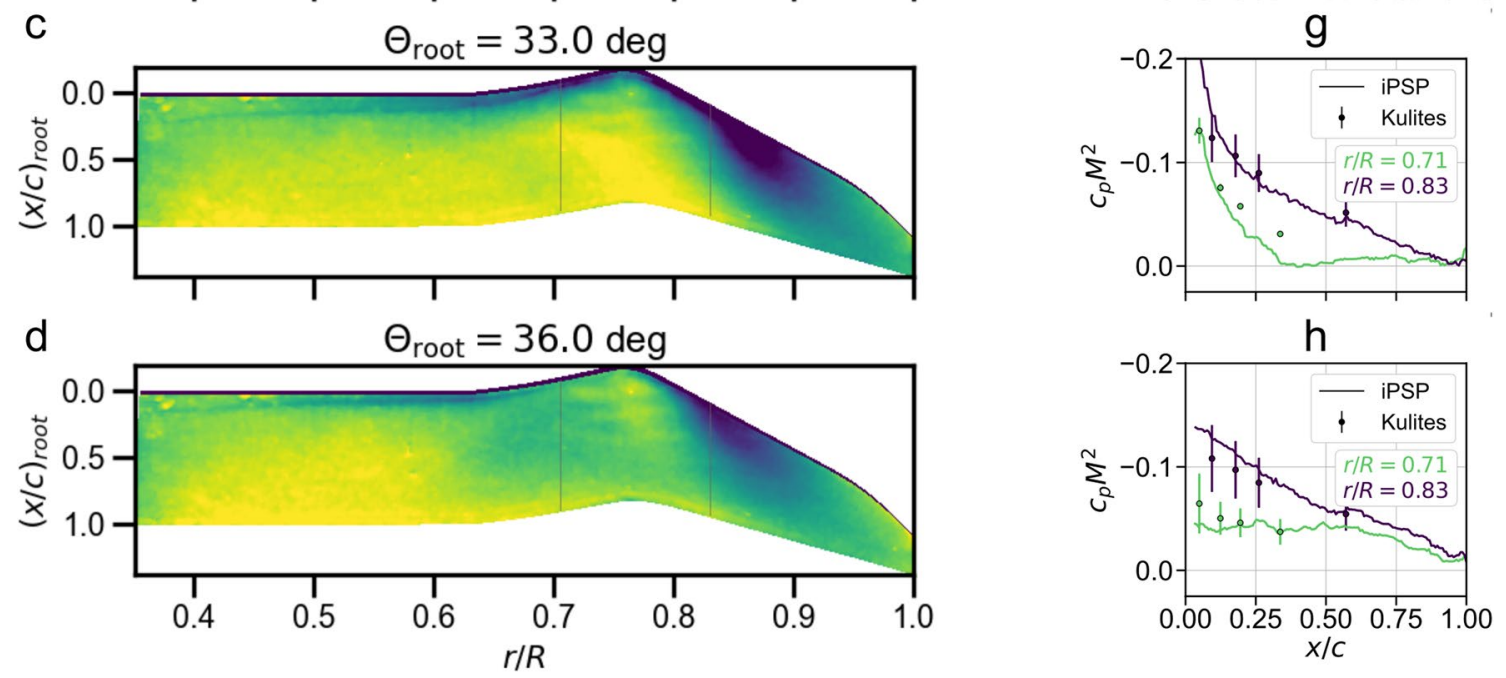

Fig. 10 Ensemble averaged surface pressure maps as $c_{p} M^{2}$ at indicated collective-pitch settings, parts (a-d), and corresponding pressure coefficients against streamwise coordinate $x / c$ for iPSP and pressure transducers at $r / R=0.71$ and $r / R=0.83$, (e-h)

At $\bar{\Theta}_{\text {root }}=24 \mathrm{deg}$ and $25 \mathrm{deg}$ the $\sigma c_{p}$ readings are enhanced by a factor of 5 in Fig. 10e, f. For these two cases at $r / R=0.83$ the pressure fluctuations are especially high for the readings at the two most upstream locations $(x / c=0.094 ; 0.178)$, which flank a kink in the pressure distribution measured by iPSP and which marks the pressure rise due to the laminar-to-turbulent boundary-layer transition (Popov et al. 2008). The phenomenon was similarly observed in PSP rotor data in the preceding campaign (Weiss et al. 2017), where the findings from PSP results and pressure transducers were supported with boundarylayer transition measurements using temperature-sensitive paint and 2D numerical computations. Additionally, at $\bar{\Theta}_{\text {root }}=25 \mathrm{deg}$ and $[r / R ; x / c]=[0.71 ; 0.195]$ the $\sigma c_{p}$ value is distinctively larger than for the adjacent unsteady pressure transducers, and the position coincides with the comparatively large positive pressure gradient distinguishable in the iPSP result, therefore marking the position of the boundary-layer transition as defined by using the established $\sigma c_{p}$ methodology (Gardner and Richter 2015; Richter et al. 2016). A close examination of the iPSP surface results reveals a dim line (highlighted in Fig. 10b) marking increased contrast between darker blue and lighter green colours across the blade span. At $\bar{\Theta}_{\text {root }}=25$ deg the line coincides with the identified transition position at $[r / R ; x / c]=[0.71 ; 0.195]$ and therefore probably marks the boundary-layer transition line, which diminishes within the suction peak at outboard radii beyond the apex and which moves successively upstream as the root pitch angle increases. It should be noted that an equivalent evaluation of the $\sigma c_{p}$ peak using the 128 single-shot pressure maps did not reveal detectable increased fluctuation levels at the identified line patterns in Fig. 10, since the fluctuation magnitudes are of a similar magnitude to measurement noise.

All results presented up to her could be measured using standard PSP. The benefit in using iPSP will be discussed 
in the next section and shows the difference to the 2017 paper.

\subsection{Cyclic-pitch cases}

\subsubsection{Test case C1}

In Fig. 11, surface pressure maps of test case $\mathrm{C} 1$ are presented for five different pitch phases, and results from iPSP measurements on the left are compared to the numerical solutions ("FLOWer") on the right using the same indicated colour map.

The iPSP results display averages of 45 phase-consecutive single-shot results corresponding to $\approx 2.8 \%$ of the pitch cycle or a blade revolution of $10 \mathrm{deg}$ azimuth, and the instantaneous numerical results include the surface streamlines of the numerical solution. Overall, the pressure topology of the numerical solution is remarkably similar to the measured iPSP result. Both results comprise similar features as previously discussed with respect to the collective-pitch cases in Fig. 10. Besides the comparable curvature of contour lines, the low-pressure region resulting from the leadingedge separation of a vortex emanating from the blade tip is similarly distinguishable from both, iPSP and numerical results. It is first visible at $t f_{\text {rotor }}=0.35$ in the figure, then progressively moves inboard during upstroke at higher pitch angles ( $t f_{\text {rotor }}=0.46$ and 0.50 ) and back towards the blade tip during downstroke $\left(t f_{\text {rotor }}=0.70\right)$. The diverging surface streamlines in the numerical solution outboard of the apex clearly support the assumed movement of the separated flow region within the pitch cycle. Note that FLOWer additionally detects separated flow close to the blade trailing edge and inboard of the apex. Closer examination (not shown here) revealed that this results from a small and shallow separation bubble induced by the adverse pressure gradient close to the trailing edge. A detailed comparison between the pressure topology from iPSP and FLOWer for the results in Fig. 11 at $0.35 \leq t f_{\text {rotor }} \leq 0.50$ reveals that the low-pressure "valley" emanating from the blade tip is consistently further inboard in the numerical solution as compared to the experiment. The finding indicates a slightly earlier separation of the leading-edge vortex in the numerical solution which also lasts slightly longer than indicated by the iPSP results.

A more detailed comparison between the experimental results from iPSP and pressure transducers and the numerical solution is provided in Fig. 12a-d.

The graphs display the streamwise pressure coefficients at four selected phase positions and at three colour-coded radial positions. The iPSP errorbar size in Fig. 12a is drawn equivalently to the description with respect to Fig. 10e. At $r / R=0.83$ the phase-consecutive iPSP average corresponding to $10 \mathrm{deg}$ azimuth (iPSP $\overline{10^{\circ}}$ ) is complemented by a single-shot result (iPSP single $)$. During upstroke at $t f_{\text {rotor }}=0.35$ (see Fig. 12a) and during downstroke at $t f_{\text {rotor }}=0.70$ (see Fig. 12d), the unsteady pressure transducer readings coincide with the numerical results within the experimental error bar sizes and both trends are matched by iPSP results with maximum deviations of $\Delta c_{p} M^{2} \approx 0.02$ at $t f_{\text {rotor }}=0.70$ and $r / R=0.52$. The same holds for the graphs at $t f_{\text {rotor }}=0.46$ and 0.50 at the two inboard radii with an exception at $r / R=0.83$. In Fig. $12 \mathrm{~b}$ at $t f_{\text {rotor }}=0.46$ the numerical result indicates premature stall onset, i.e. leading-edge separation which is characterized by the breakdown of the suction peak and the pressure plateau between $0.1 \leq x / c \leq 0.2$. The low pressure plateau in the simulation convects downstream very rapidly (see Fig. 12c between $0.25 \leq x / c \leq 0.5$ ), which is characteristic of dynamic stall. However, due to the chaotic nature of this process which produces large cycle-to-cycle
Fig. 11 Surface pressure maps resulting from iPSP measurements and numerical computations as $c_{p} M^{2}$ at different phases of unsteady test case $\mathrm{C} 1$

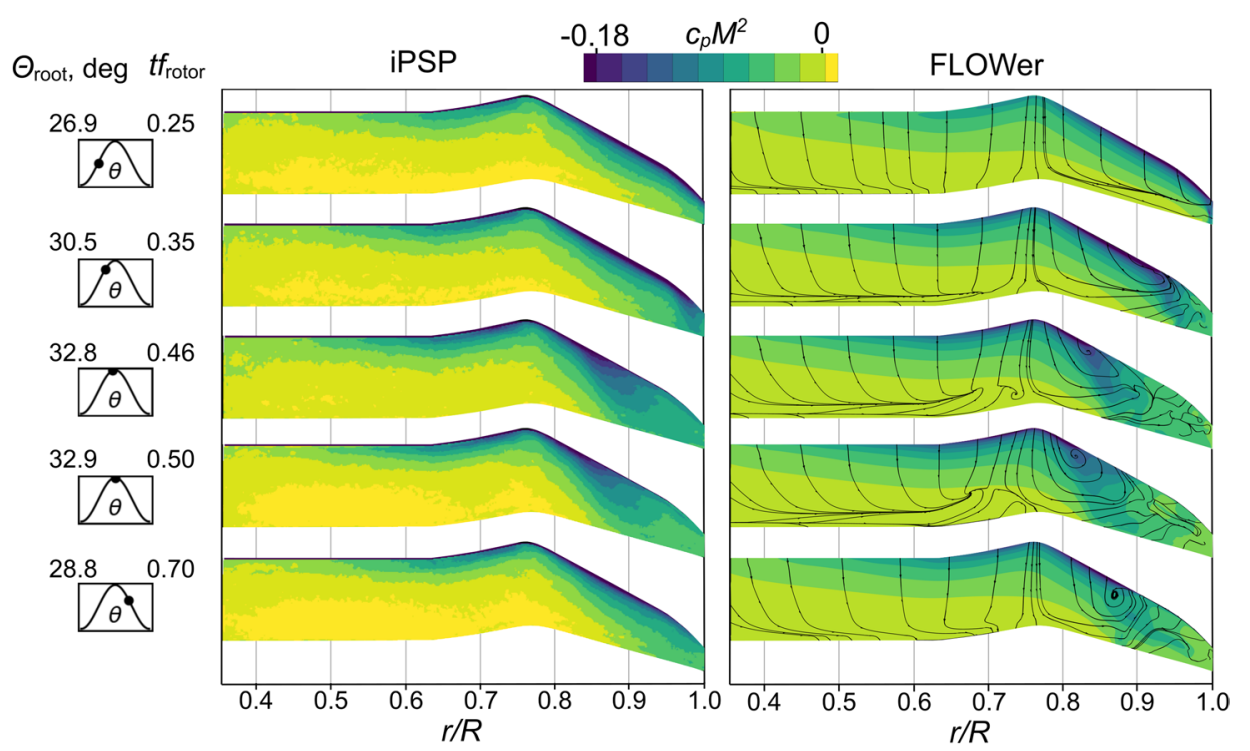


Fig. 12 Pressure coefficient $c_{p} M^{2}$ against streamwise coordinate $x / c$. at different radial positions $r / R$ and pitch phases $t f_{\text {rotor }}$. Comparison between iPSP data averaged over $10 \mathrm{deg}$ azimuth, single-shot iPSP data at $r / R=0.83$, numerical results (FLOWer) and unsteady pressure transducer measurements (Kulites)

variations this instantaneous numerical result cannot be observed in the phase-averaged data of the unsteady pressure transducers. Still, the large cycle-to-cycle variations are indicated by the increased error bar sizes in the pressure transducer results. It is known that all eddy-viscosity turbulence models have difficulties in predicting the onset and extent of flow separation (Blazek 2015). However, regarding the single-shot iPSP result at $t f_{\text {rotor }}=0.46$, it might even be possible to find a similar pressure distribution at a slightly later point in the pitch cycle or at a radial station slightly more in- or outboard. At $t f_{\text {rotor }}=0.50$ the separation observed in the numerical result is qualitatively matched by the instantaneous single-shot result from iPSP, at least for $x / c<0.3$. Further downstream, the iPSP single-shot result and the numerical prediction diverge before they rejoin towards the blade trailing edge. The finding expresses again the unsteady behaviour of the flow at $r / R=0.83$ and at the maximumpitch setting. It is also interesting to note that the behaviour is filtered by the phase-consecutive iPSP average, although the averaging interval is quite small $\left(\Delta t f_{\text {rotor }}=0.028\right)$.

Pressure fluctuations were evaluated from iPSP results by calculating the running standard deviation of surface pressures with a window size of 45 phase-consecutive singleshot results corresponding to $\Delta t f_{\text {rotor }}=0.028$. In Fig. 13, the pressure fluctuation images are normalized by the ambient pressure as $\sigma_{p} / p_{\infty}$ and mapped on a disk, where the start of the pitch cycle at minimum-pitch angle corresponds to the 3 o'clock position with the blade rotating counter-clockwise. The displayed colour bar range was chosen such that relatively higher pressure fluctuation levels can be distinguished in dark from the base level of fluctuations, which is displayed in light grey. The base level includes measurement noise as well as pressure fluctuations caused by different pitch angles during the considered phase period and also cycle-to-cycle variations. Recall that each of the processed phase-consecutive images were acquired at different rotation cycles.

In Fig. 13, the regions on the blade with distinguishably increased pressure fluctuations are influenced by large detached vortical structures and thus indicate stalled flow. The figure can therefore be interpreted as a stall map of test case $\mathrm{C} 1$. Similar ideas were implemented by Gardner et al. (2016) and Raffel et al. (2017), who deduced stall maps using the standard deviation of thermal difference images of a $2 \mathrm{D}$ pitching airfoil and a rotating blade in a dynamic-stall condition. The experimental results in Fig. 13 are complemented by numerical flow visualizations at four
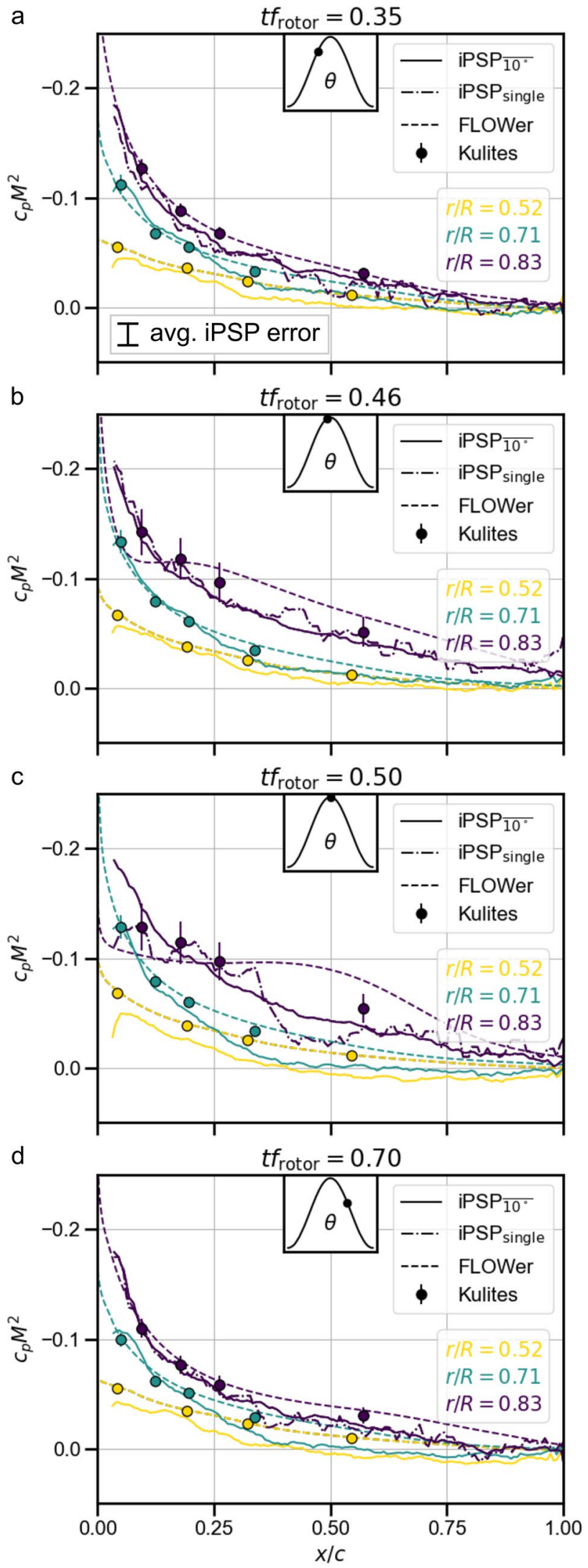
Fig. 13 Stall map of test case $\mathrm{C} 1$ displaying relative pressure fluctuation levels on rotor blade at sixteen phase positions and numerical flow visualizations (from FLOWer) showing isosurfaces of the $\lambda_{2}$ criterion colour coded by the ratio of eddy viscosity to laminar viscosity

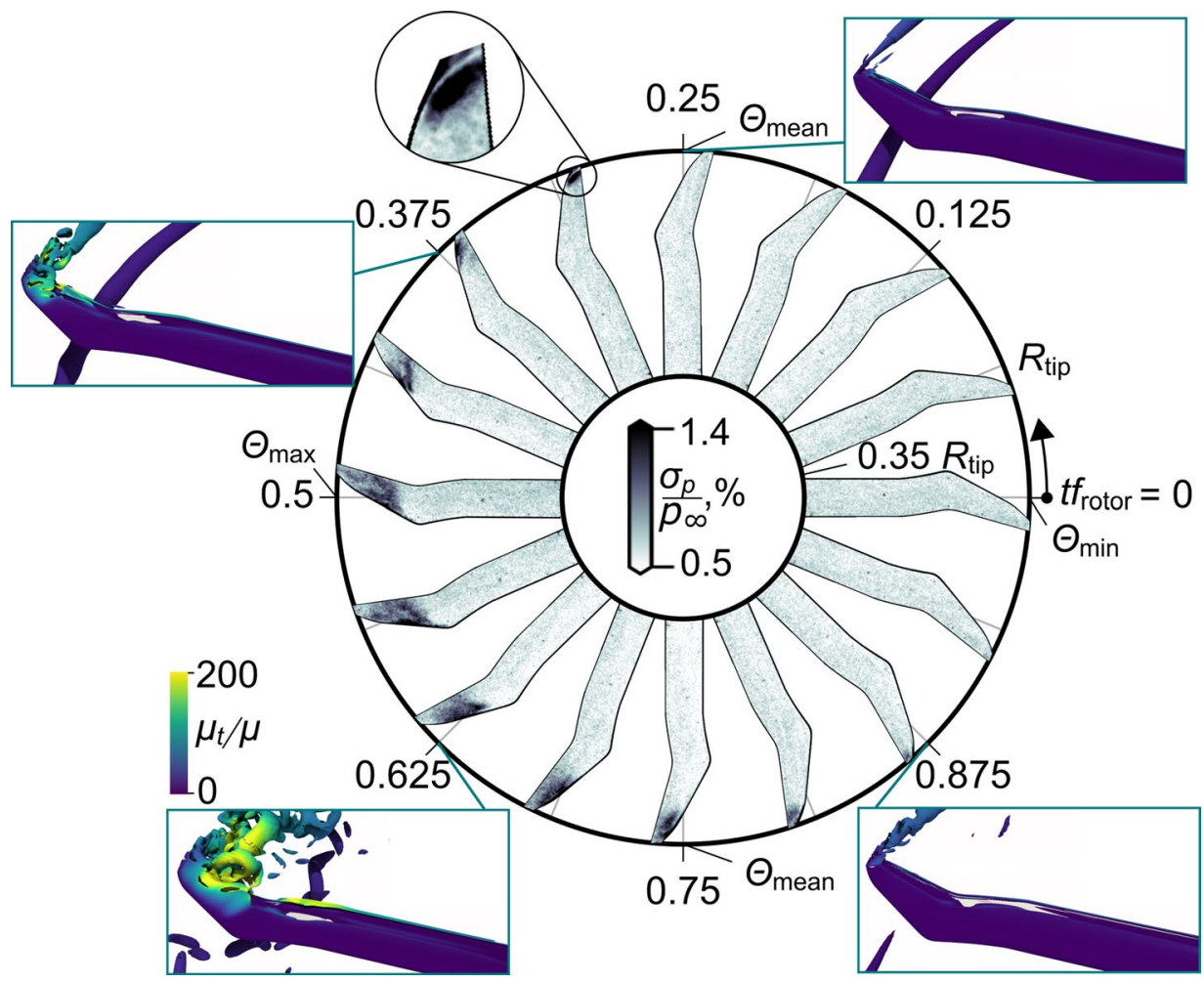

representative phase positions $\left(t f_{\text {rotor }}=0.25 ; 0.375 ; 0.625\right.$ and 0.875$)$. The visualizations display isosurfaces of the $\lambda_{2}$ criterion (Jeong and Hussain 1995), colour-coded by the ratio of eddy viscosity to laminar viscosity $\mu_{t} / \mu$, which is especially large for detached flow.

Between $0 \leq t f_{\text {rotor }} \leq 0.25$, no increased pressure fluctuations are detectable. The flow is attached to the blade, and only the blade tip vortex evolves downstream as displayed by the visualization at $t f_{\text {rotor }}=0.25$. However, at $t f_{\text {rotor }}=0.313$, the tip vortex detaches close to the blade tip and leaves a footprint with increased pressure fluctuations. The incipient stalled flow area then grows inboard and reaches its maximum during $0.5<t f_{\text {rotor }}<0.625$ and after the pitch angle reaches $\Theta_{\max }$. The corresponding flow visualization at $t f_{\text {rotor }}=0.625$ shows large vortical structures shedding from outboard of the blade apex. The numerical result further underlines that the stalled flow area as indicated by the iPSP results never reaches inboard of the blade apex but decreases during downstroke until it can hardly be detected anymore at around $t f_{\text {rotor }}=0.875$. Note also that when stalled flow can be distinguished in the iPSP results, the pressure fluctuations are higher close to the leading edge as compared to further downstream, e.g. at $t f_{\text {rotor }}=0.625$, which underlines that the separation originates at the blade leading edge as indicated by the numerical visualizations. The asymmetrical development of the stalled flow area with respect to the maximumpitch angle at $t f_{\text {rotor }}=0.5$ and the resulting hysteresis originate from the unsteadyness of the circulation of the bound vortex and the resulting effect of the vortex density in the wake. This phenomenon was first described by Theodorsen's lift deficiency function (see Theodorsen 1935). An interesting feature is visible close to the blade tip at $t f_{\text {rotor }}=0.313$. As highlighted in the detailed view, an oblique white stripe with low pressure fluctuation levels crosses the stalled dark area at the blade tip. It is assumed that it resembles the footprint of the vortex system which detaches from the leading edge of the backward-swept blade tip and convects downstream close to the blade surface, as similarly seen in the flow visualization at $t f_{\text {rotor }}=0.375$. Note that the vortical structure has to be of a high coherence, since the pressure fluctuation image results from 45 different blade revolutions.

\subsubsection{Test case $\mathrm{C} 2$}

An equivalent stall map as discussed above for test case $\mathrm{C} 1$ is presented for test case $\mathrm{C} 2$ in Fig. 14, where the same pitch amplitude was applied as in $\mathrm{C} 1$ but at a collective-pitch angle which is 6 deg larger.

The stall map is complemented by flow visualizations (obtained with TAU and taken from Kaufmann et al. (2020)), showing the three-dimensional dynamic stall behaviour for test case $\mathrm{C} 2$ by means of isosurfaces of the $\lambda_{2}$ criterion, which were colour-coded by the velocity magnitude of the component $w$ normal to the rotor disk. In contrast to the test case presented above, the blade tip exhibits detached flow at all times for test case $\mathrm{C} 2$. Additionally, the flow even 
Fig. 14 Stall map of test case $\mathrm{C} 2$ displaying relative pressure fluctuation levels on rotor blade at sixteen phase positions and flow visualizations from numerical computations (Kaufmann et al. 2020) showing isosurfaces of the $\lambda_{2}$ criterion colour coded by the velocity component perpendicular to the rotor plane

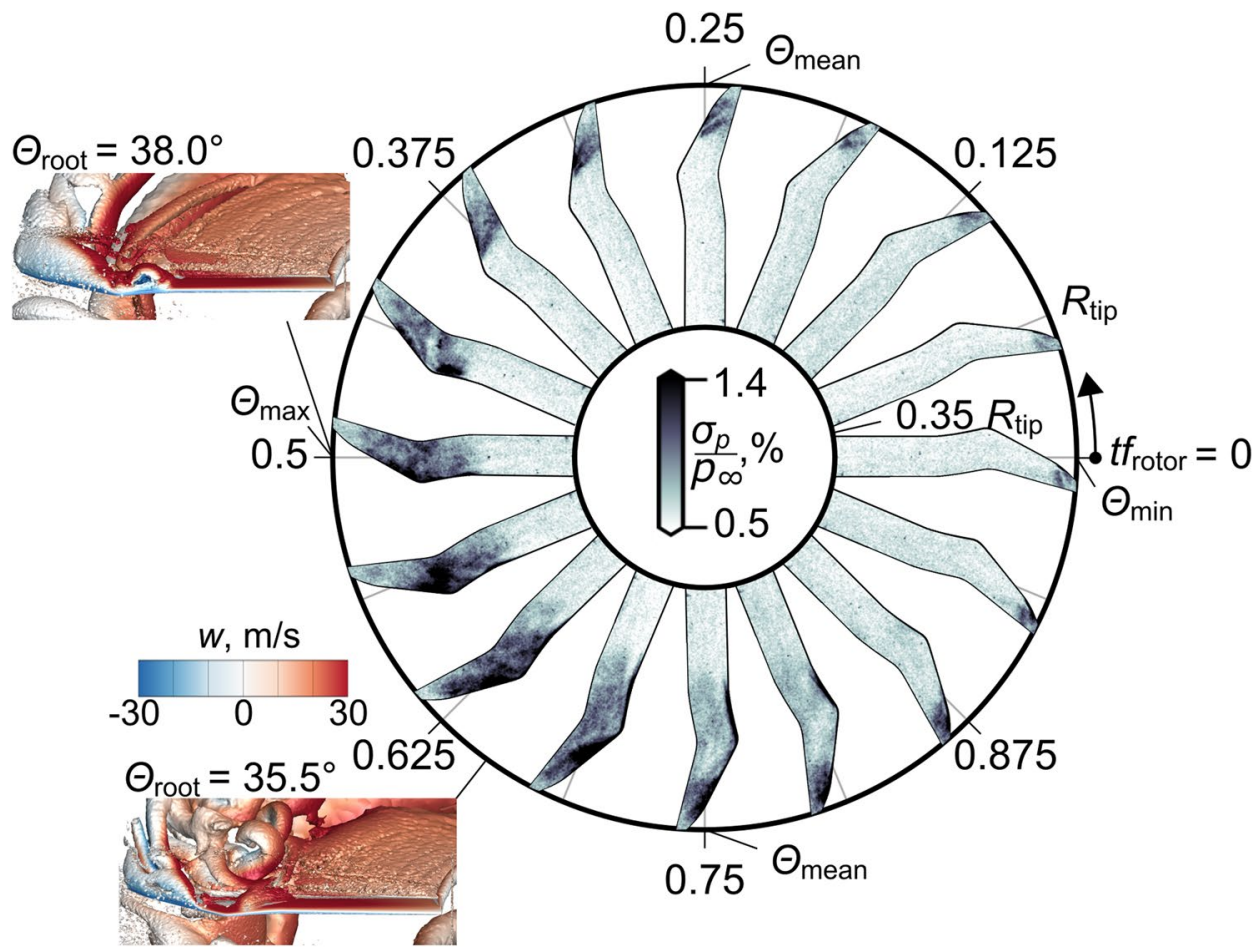

stalls inboard of the apex, which is first distinguishable at $t f_{\text {rotor }}=0.438$ in Fig. 14. The stalled flow area reaches a maximum at about $0.5<t f_{\text {rotor }}<0.625$. The iPSP finding is supported by the flow visualizations from the numerical solution at $t f_{\text {rotor }}=0.5$ and around $t f_{\text {rotor }}=0.63$, which show the formation of an omega-shaped vortex structure just inboard of the apex as a characteristic feature of three-dimensional dynamic stall as well as the growth of the stalled area further inboard. Note that the stall behaviour outboard and inboard of the apex differs significantly. Outboard of the apex, the stalled area gradually increases and decreases during the pitch cycle. Inboard of the apex, stall onset seems to occur rather abruptly during upstroke at $t f_{\text {rotor }} \approx 0.42$, and the end of stall during downstroke at $t f_{\text {rotor }} \approx 0.75$ is accompanied by large pressure fluctuations close to the leading edge.

In Fig. 15, the pressure fluctuations calculated from iPSP results are compared to the cycle-to-cycle fluctuations $\sigma c_{p}$ recorded with the pressure transducers at three exemplary positions inboard of the apex $(r / R=0.71)$, on the apex $(r / R=0.77)$ and outboard of it $(r / R=0.83)$. Note that the $\sigma c_{p}$ signal denotes the phase-locked standard deviation from $\approx 3200$ blade revolutions. For this comparison the pressure fluctuations from iPSP were averaged over a $3 \times 3$ kernel on the $3 \mathrm{D}$ blade grid, centred at the indicated pressure transducer position and corresponding to an area of $\approx 1 \mathrm{~mm}^{2}$. The area is slightly larger than the pressure tap with a diameter of $0.3 \mathrm{~mm}$ and yet reasonable for a comparison. The three colour-coded graphs in Fig. 15

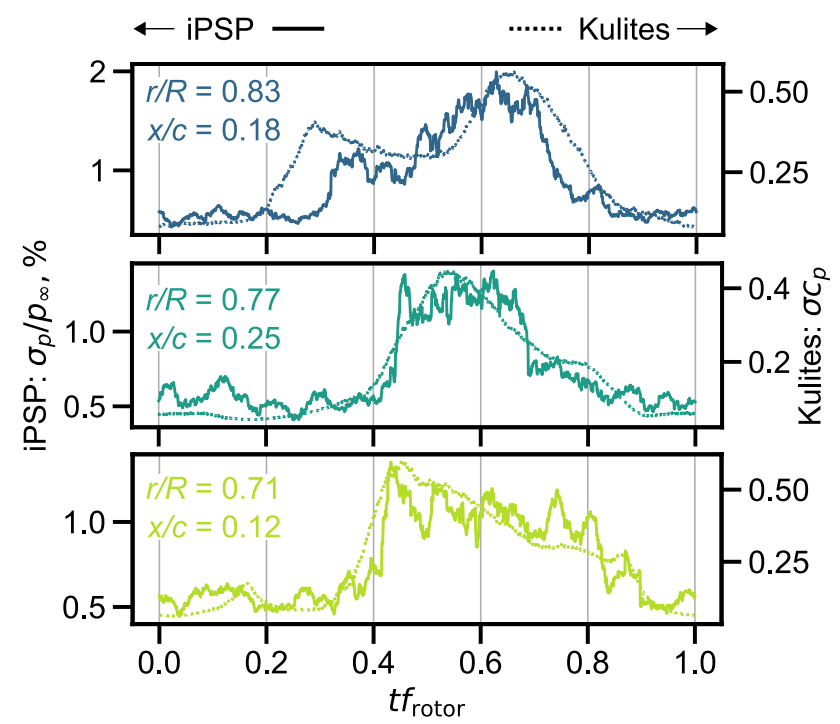

Fig. 15 Pressure fluctuation results at three positions on the blade for test case C2. The iPSP signal displays the normalized standard deviation of surface pressures from 45 phase consecutive images of different revolutions, i.e. $\Delta t f_{\text {rotor }}=0.028$. The pressure transducer signal (Kulites) displays the cycle-to-cycle standard deviation of $c_{p}$ from $\approx$ 3200 revolutions

correspond to the indicated radial positions. At $r / R=0.71$ and $r / R=0.77$ the graphs for iPSP and pressure transducers indicate nearly identical phase positions with a significant increase of the measured pressure fluctuations, which corresponds to stall onset at $t f_{\text {rotor }}=0.4$ and $t f_{\text {rotor }}=0.45$, 
respectively. During downstroke the pressure fluctuations at these two radial positions decrease gradually and quite similarly for both measurement techniques until the bottom level of fluctuations is reached again, and flow is assumed to be attached at $t f_{\text {rotor }} \approx 0.9$. At $r / R=0.83$, the pressure increase is detected a little sooner by the pressure transducer than by iPSP $\left(\Delta t f_{\text {rotor }} \approx 0.1\right)$, and the decrease is detected a little later at $\left(\Delta t f_{\text {rotor }} \approx 0.07\right)$, but the qualitative evolution of the two pressure fluctuation signals is still remarkably similar. A possible reason for the deviations at $r / R=0.83$ between the pressure transducer and iPSP result might be that both signals are recorded on two different blades, which should nominally have the same shape and twist but which naturally differ slightly due to manufacturing tolerances.

\section{Conclusions}

An optimized pressure-sensitive paint measurement system was successfully applied to investigate dynamic stall on double-swept rotor blades at blade tip Mach and Reynolds numbers of $M_{\text {tip }}=0.282-0.285$ and $R e_{\text {tip }}=5.84-5.95 \times 10^{5}$ at DLR's rotor test facility, RTG. The measurement system components were presented, including the employed iPSP sensor and with a special emphasis on the improved version of a fast double-shutter camera, which was previously used in order to eliminate the problem of image blur when applying the single-shot lifetime technique to PSP measurements on fast-rotating blades. The global unsteady pressure maps were acquired across the outer $65 \%$ of the blade and complemented by fast-response pressure tranducer readings at several radial positions. Experimental results were further compared to numerical simulations using the flow solver FLOWer and DLR's TAU code. The main outcomes of the presented study can be summarized as follows:

1. The quality of resulting surface pressure maps is significantly enhanced by the improved camera acquisition mode due to an increase of the SNR by a factor of $\sqrt{2}$ when using the optimized acquisition mode presented in this study.

2. A comparison between iPSP results and pressure transducer readings across the blade span revealed a mean absolute deviation of $\approx 750 \mathrm{~Pa}$ when considering all 37 data points with only collective-pitch angles. The results of collective-pitch cases allowed for the extraction of flow features as the footprint of a detached leading-edge vortex on the outer backward-swept part of the blade as well as the kink in the pressure distribution due to the laminar-to-turbulent boundary-layer transition.

3. Excellent qualitative and quantitative agreement between pressure transducer readings and numerical computations (with FLOWer) was achieved in the investigated cyclic-pitch case. The experimental surface pressure topology from iPSP showed excellent qualitative agreement to the numerical solution with similar features as observed for the collective-pitch settings. Only minor differences between iPSP and numerical unsteady surface pressure results occurred with respect to the time instants of flow separation and reattachment.

4. Pressure fluctuation data deduced from iPSP results allow for identifying stall maps with confined regions corresponding to stalled flow as a function of the pitch phase as well as the extraction of interesting flow features. The experimental results are well supported by corresponding flow visualizations from FLOWer and TAU computations. The findings revealed that outboard of the blade apex the stalled flow area increases and decreases more gradually along the pitch cycle, as opposed to inboard positions of the apex. Here, stall onset occurs later in the pitch cycle at larger pitch angles, and reattachment occurs earlier and at larger pitch angles than outboard of the apex. Also, incipient stall is more spontaneous inboard of the apex as opposed to outboard of it.

5. Data extracted from the iPSP stall map showed good agreement to unsteady pressures measured by pressure transducers.

6. The study presents the first comprehensive iPSP data set on a rotor under cyclic-pitch conditions at all pitch phases and its findings are supported by the results of numerical computations.

Acknowledgements The support of Markus Krebs in preparing the experiment at the RTG and of Florian Philipp (both of DLR Göttingen) in supporting image acquisition with a handy frame grabber software is gratefully acknowledged. The authors also thank Daisuke Yorita for carrying out the measurements of the luminescence decay curves, Carsten Fuchs for his support in foil application on the rotor blade and in assisting the 3D measurements of the dot markers which were carried out by Daniel Drössler (all of DLR Göttingen) whose support is evenly acknowledged.

Funding Open Access funding enabled and organized by Projekt DEAL.

Open Access This article is licensed under a Creative Commons Attribution 4.0 International License, which permits use, sharing, adaptation, distribution and reproduction in any medium or format, as long as you give appropriate credit to the original author(s) and the source, provide a link to the Creative Commons licence, and indicate if changes were made. The images or other third party material in this article are included in the article's Creative Commons licence, unless indicated otherwise in a credit line to the material. If material is not included in the article's Creative Commons licence and your intended use is not permitted by statutory regulation or exceeds the permitted use, you will need to obtain permission directly from the copyright holder. To view a copy of this licence, visit http://creativecommons.org/licenses/by/4.0/. 


\section{References}

Bickel G, Häusler G, Maul M (1985) Triangulation with expanded range of depth. Opt Eng 24(6):975-977. https://doi.org/10.1117/ 12.7973610

Blazek J (2015) Chapter 7-turbulence modeling. In: Computational fluid dynamics: principles and applications, 3rd ed. ButterworthHeinemann, Oxford, pp 213-252. https://doi.org/10.1016/B9780-08-099995-1.00007-5

Bousman WG (1998) A qualitative examination of dynamic stall from flight test data. J Am Helicopter Soc 43(4):279-295. https://doi. org/10.4050/JAHS.43.279

Disotell KJ, Peng D, Juliano TJ, Gregory JW, Crafton JW, Komerath NM (2014) Single-shot temperature- and pressure-sensitive paint measurements on an unsteady helicopter blade. Exp Fluids 55(2):1671. https://doi.org/10.1007/s00348-014-1671-2

Egami Y (2020) Microscopic measurements on DLR's time-resolved pressure-sensitive paint coating, private communication. Dept. of Mechanical Engineering, Aichi Institute of Technology, Japan

Gardner AD, Richter K (2015) Boundary layer transition determination for periodic and static flows using phase-averaged pressure data. Exp Fluids 56(6):119-131. https://doi.org/10.1007/ s00348-015-1992-9

Gardner AD, Wolf CC, Raffel M (2016) A new method of dynamic and static stall detection using infrared thermography. Exp Fluids 57(9):149. https://doi.org/10.1007/s00348-016-2235-4

Geisler R (2014) A fast double shutter system for CCD image sensors. Meas Sci Technol 25(2):025404. https://doi.org/10.1088/09570233/25/2/025404

Geisler R (2017a) A fast double shutter for CCD-based metrology. In: Shiraga H, Etoh TG (eds) Selected papers from the 31st international congress on high-speed imaging and photonics, SPIE, Osaka, Japan, vol 10328, p 1032809. https://doi.org/10.1117/12. 2269099

Geisler R (2017) A fast multiple shutter for luminescence lifetime imaging. Meas Sci Technol 28(9):095403. https://doi.org/10.1088/ 1361-6501/aa7aca

Goerttler A, Braukmann JN, Schwermer T, Gardner AD, Raffel M (2018) Tip-vortex investigation on a rotating and pitching rotor blade. J Aircr 55(5):1792-1804. https://doi.org/10.2514/1.C0346 93

Gregory J, Sakaue H, Sullivan J (2002) Unsteady pressure measurements in turbomachinery using porous pressure sensitive paint. In: 40th AIAA aerospace sciences meeting \& exhibit, AIAA 20020084, American Institute of Aeronautics and Astronautics. https:// doi.org/10.2514/6.2002-84

Gregory JW, Kumar P, Peng D, Fonov S, Crafton J, Liu T (2009) Integrated optical measurement techniques for investigation of fluidstructure interactions. In: 39th AIAA fluid dynamics conference, San Antonio, TX, USA, AIAA 2009-4044. https://doi.org/10. 2514/6.2009-4044

Gritskevich MS, Garbaruk AV, Menter FR (2013) Fine-tuning of DDES and IDDES formulations to the k-ar stress transport model. https://doi.org/10.1051/eucass/201305023

Gößling J, Ahlefeldt T, Hilfer M (2020) Experimental validation of unsteady pressure-sensitive paint for acoustic applications. Exp Thermal Fluid Sci 112:109915. https://doi.org/10.1016/j.expth ermflusci.2019.109915

Jeong J, Hussain F (1995) On the identification of a vortex. J Fluid Mech 285:69-94. https://doi.org/10.1017/S0022112095000462

Jiao L, Liu X, Shi Z, Zhang W, Liang L, Chen X, Zhao Q, Peng D, Liu Y (2020) A two-dimensional temperature correction method for pressure-sensitive paint measurement on helicopter rotor blades. Exp Fluids 61(4):104. https://doi.org/10.1007/s00348-020-2929-5
Johnson W (1998) Rotorcraft aerodynamics models for a comprehensive analysis. In: Proceedings of the 54th annual forum of the American Helicopter Society, American Helicopter Society. http:// johnson-aeronautics.com/documents/CIIaerodynamics.pdf

Juliano TJ, Kumar P, Peng D, Gregory JW, Crafton J, Fonov S (2011) Single-shot, lifetime-based pressure-sensitive paint for rotating blades. Meas Sci Technol 22(8):085403. https://doi.org/10.1088/ 0957-0233/22/8/085403

Kaufmann K, Müller MM, Gardner AD (2020) Dynamic stall computations of double-swept rotor blades. In: Dillmann A, Heller G, Krämer E, Wagner C, Tropea C, Jakirlić S (eds) New results in numerical and experimental fluid mechanics XII: contributions to the 21st STAB/DGLR symposium Darmstadt, Germany, 2018, Springer International Publishing, Cham, Notes on Numerical Fluid Mechanics and Multidisciplinary Design, vol 142, pp 351-361. https://doi.org/10.1007/978-3-030-25253-3_34

Klein C, Engler RH, Henne U, Sachs WE (2005) Application of pressure-sensitive paint for determination of the pressure field and calculation of the forces and moments of models in a wind tunnel. Exp Fluids 39(2):475-483. https://doi.org/10.1007/ s00348-005-1010-8

Leishman JG (2006) Principles of helicopter aerodynamics, 2nd edn. Cambridge University Press, Cambridge

Letzgus J, Gardner AD, Schwermer T, Keßler M, Krämer E (2019) Numerical investigations of dynamic stall on a rotor with cyclic pitch control. J Am Helicopter Soc 64(1):1-14. https://doi.org/ 10.4050/JAHS.64.012007

Letzgus J, Keßler M, Krämer E (2020) Simulation of dynamic stall on an elastic rotor in high-speed turn flight. J Am Helicopter Soc 65(2):1-12. https://doi.org/10.4050/JAHS.65.022002

Liu T, Sullivan JP, Asai K, Klein C, Egami Y (2021) Pressure and temperature sensitive paints. Springer International Publishing. https://doi.org/10.1007/978-3-030-68056-5

McLachlan B, Bell J (1995) Pressure-sensitive paint in aerodynamic testing. Exp Thermal Fluid Sci 10(4):470-485. https://doi.org/ 10.1016/0894-1777(94)00123-P

Müller MM, Schwermer T, Mai H, Stieg C (2018) Development of an innovative double-swept rotor blade-tip for the rotor test facility Goettingen. In: DLRK 2018 - Deutscher Luft- und Raumfahrtkongress, DGLR - Deutsche Gesellschaft für Luft- und Raumfahrt - Lilienthal-Oberth e.V., Friedrichshafen. https://elib.dlr. de/122702/

Ondrus V (2020) Development of pressure-sensitive paint to measure unsteady flow phenomena, private communication. University of Applied Sciences Münster, Germany, Department of Chemical Engineering

Pandey A, Gregory JW (2018) Iterative blind deconvolution algorithm for deblurring a single PSP/TSP image of rotating surfaces. Sensors 18(9):1-22. https://doi.org/10.3390/s18093075

Peng D, Liu Y (2019) Fast pressure-sensitive paint for understanding complex flows: from regular to harsh environments. Exp Fluids 61(1):8. https://doi.org/10.1007/s00348-019-2839-6

Popov AV, Botez RM, Labib M (2008) Transition point detection from the surface pressure distribution for controller design. J Aircr 45(1):23-28. https://doi.org/10.2514/1.31488

Puklin E, Carlson B, Gouin S, Costin C, Green E, Ponomarev S, Tanji H, Gouterman M (2000) Ideality of pressure-sensitive paint. I. Platinum tetra(pentafluorophenyl)porphine in fluoroacrylic polymer. J Appl Polym Sci 77(13):2795-2804. https://doi.org/10.1002/ 1097-4628(20000923)77:13<2795::AID-APP1>3.0.CO;2-K

Raddatz J, Fassbender JK (2005) Block structured Navier-Stokes solver FLOWer. In: Kroll N, Fassbender JK (eds) MEGAFLOWnumerical flow simulation for aircraft design, Springer Berlin Heidelberg, Berlin, Heidelberg, pp 27-44. https://doi.org/10. 1007/3-540-32382-1_2 
Raffel M, Heineck JT (2014) Mirror-based image derotation for aerodynamic rotor measurements. AIAA J 52(6):1337-1341. https:// doi.org/10.2514/1.J052836

Raffel M, Gardner AD, Schwermer T, Merz CB, Weiss A, Braukmann J, Wolf CC (2017) Rotating blade stall maps measured by differential infrared thermography. AIAA J 55(5):1-4. https://doi. org/10.2514/1.J055452

Richter K, Wolf CC, Gardner AD, Merz CB (2016) Detection of unsteady boundary layer transition using three experimental methods. In: 54th AIAA aerospace sciences meeting, San Diego, CA, USA. https://doi.org/10.2514/6.2016-1072

Ruyten W, Sellers M (2006) Improved data processing for pressuresensitive paint measurements in an industrial facility. In: 44th AIAA aerospace sciences meeting and exhibit, Reno, NV, AIAA 2006-1042. https://doi.org/10.2514/6.2006-1042

Schairer E, Mehta R, Olsen M, Hand L, Bell J, Whittaker P, Morgan D (1998) The effects of thin paint coatings on the aerodynamics of semi-span wings. In: 36th AIAA aerospace sciences meeting and exhibit, no. 0 in aerospace sciences meetings, American Institute of Aeronautics and Astronautics, Reno, NV, USA. https://doi.org/ $10.2514 / 6.1998-587$

Schlichting H, Gersten K (2017) Boundary-layer theory, 9th edn. Springer, Berlin Heidelberg. https://doi.org/10.1007/ 978-3-662-52919-5

Schwamborn D, Gardner AD, von Geyr H, Krumbein A, Lüdeke H, Stürmer A (2008) Development of the tau-code for aerospace applications. In: Proceedings of the 50th international conference on aerospace science and technology, Indian National Aerospace Lab. (NAL), Bangalore, India, Paper IT-13. https://elib.dlr.de/ 55519/1/INCAST_Schwamborn.pdf

Schwermer T, Richter K, Raffel M (2016) Development of a rotor test facility for the investigation of dynamic stall. In: Dillmann A, Heller G, Krämer E, Wagner C, Breitsamter C (eds) New results in numerical and experimental fluid mechanics $\mathrm{X}$ : contributions to the 19th STAB/DGLR symposium Munich, Germany, 2014, Springer International Publishing, Cham, Notes on Numerical Fluid Mechanics and Multidisciplinary Design, vol 132, pp 663 673. https://doi.org/10.1007/978-3-319-27279-5_58

Schwermer T, Gardner AD, Raffel M (2019) A novel experiment to understand the dynamic stall phenomenon in rotor axial flight. J Am Helicopter Soc 64(1):1-11. https://doi.org/10.4050/JAHS. 64.012004

Scroggin AM, Slamovich EB, Crafton JW, Lachendro N, Sullivan JP (1999) Porous polymer/ceramic composites for luminescence-based temperature and pressure measurement. MRS Online Proc Libr 560(1):347-352. https://doi.org/10.1557/ PROC-560-347

Sugimoto T, Sugioka Y, Numata D, Nagai H, Asai K (2017) Characterization of frequency response of pressure-sensitive paints. AIAA J 55(4):1460-1464. https://doi.org/10.2514/1.J054985

Theodorsen T (1935) General theory of aerodynamic instability and the mechanism of flutter. NACA Technical Report NACA-ARR-1935 superseded by NACA-TR-496, National Advisory Committee for Aeronautics, Langley Aeronautical Lab, Langley Field, VA, USA. https://ntrs.nasa.gov/api/citations/19930090935/downloads/19930 090935.pdf

Watkins AN, Leighty BD, Lipford WE, Goodman KZ, Crafton J, Gregory JW (2016) Measuring surface pressures on rotor blades using pressure-sensitive paint. AIAA J 54(1):206-215. https://doi.org/ 10.2514/1.J054191

Weiss A, Geisler R, Schwermer T, Yorita D, Henne U, Klein C, Raffel M (2017) Single-shot pressure-sensitive paint lifetime measurements on fast rotating blades using an optimized double-shutter technique. Exp Fluids 58(9):120-140. https://doi.org/10.1007/ s00348-017-2400-4

Yamauchi GK, Wadcock AJ, Heineck JT (1997) Surface flow visualization on a hovering tilt rotor blade. In: Proceedings of the AHS technical meeting on rotorcrafts acoustics and aerodynamics, AHS International, Fairfax, VA, USA

Yorita D, Klein C, Henne U, Ondrus V, Beifuss U, Hensch AK, Longo R, Guntermann P, Quest J (2018a) Successful application of cryogenic pressure-sensitive paint technique at ETW. In: 2018 AIAA aerospace sciences meeting, no. AIAA 2018-1136 in AIAA SciTech Forum, American Institute of Aeronautics and Astronautics, Kissimmee, FL, USA. https://doi.org/10.2514/6.2018-1136

Yorita D, Weiss A, Geisler R, Henne U, Klein C (2018b) Comparison of LED and LASER based lifetime pressure-sensitive paint measurement techniques. In: 2018 AIAA aerospace sciences meeting, no. AIAA 2018-1029 in AIAA SciTech Forum, American Institute of Aeronautics and Astronautics, Kissimmee, FL, USA. https://doi.org/10.2514/6.2018-1029

Publisher's Note Springer Nature remains neutral with regard to jurisdictional claims in published maps and institutional affiliations. 